\title{
Delayed Onset of Crystallinity in Ion-Containing Aqueous Nanodrops
}

\author{
Richard J. Cooper, Matthew J. DiTucci, Terrence M. Chang and Evan R. Williams* \\ Department of Chemistry, University of California, Berkeley, California, 94720-1460
}

\section{Supporting Information}




\section{Methods}

IRPD Spectroscopy. IRPD spectra are acquired using a 7.0 T Fourier-transform ion cyclotron resonance mass spectrometer that was recently upgraded from a $2.7 \mathrm{~T}$ magnet and that is coupled to a tunable infrared laser. ${ }^{1}$ Hydrated lanthanum ions are formed by nanoelectrospray ionization of a $\sim 4 \mathrm{mM}$ solution of $\mathrm{LaCl}_{3}$ (Sigma-Aldrich, St. Louis, MO). Hydrated ions are gently transferred though an electrospray ionization interface ${ }^{2}$ by electrostatic lenses into a cylindrical ion cell surrounded by a copper jacket ${ }^{3}$ that is regulated to $133 \mathrm{~K}$ by a controlled flow of liquid nitrogen. A $\sim 5$ s pulse of dry nitrogen gas aids with the trapping and thermal equilibration of the ions. The ions of interest are isolated with stored waveform inverse Fourier transform excitations. An ensemble averaging technique ${ }^{4}$ is used to increase the signal-to-noise ratios of the spectra by mass selecting distributions of precursor ions that include between 3 and 11 adjacent cluster sizes. A weighted-average cluster size is reported throughout. The massselected clusters are subsequently irradiated with tunable infrared laser light from a OPO/OPA tabletop laser system (LaserVision, Bellevue, WA) pumped by the $1064 \mathrm{~nm}$ fundamental of a Nd:YAG laser (Continuum Surelight I-10, Santa Clara, CA) at $10 \mathrm{~Hz}$ repetition rate yielding pulse energies between $\sim 1-4 \mathrm{~mJ}$ in the spectral range between $3000-3800 \mathrm{~cm}^{-1}$. Irradiation times between $0.5-1 \mathrm{~s}$ are used to dissociate the precursor ions by the sequential loss of water molecules, and photodissociation rate constants are obtained from these data. All photodissociation rate constants are corrected for frequency-dependent variations in laser power as well dissociation due to the absorption of blackbody radiation and are plotted as a function of wavelength to give IRPD spectra. Under these conditions, the absorption of one or more photons is sufficient to increase the rate of dissociation such that the IRPD spectra presented here should closely resemble linear absorption spectra. 
There are several pieces of evidence that suggest that lanthanum exists as $\mathrm{La}^{3+}$ in these clusters, and not as the separate ion pairs, $\mathrm{LaOH}^{2+}$ and $\mathrm{H}_{3} \mathrm{O}^{+}$. Unimolecular dissociation experiments demonstrate that $\mathrm{La}^{3+}\left(\mathrm{H}_{2} \mathrm{O}\right)_{n}$ clusters are stable with respect to the charge separation reaction, $\mathrm{La}^{3+}\left(\mathrm{H}_{2} \mathrm{O}\right)_{n} \rightarrow\left[\mathrm{La}(\mathrm{OH})\left(\mathrm{H}_{2} \mathrm{O}\right)_{n-2}\right]^{2+}+\mathrm{H}_{3} \mathrm{O}^{+}$for $n>17$. . $^{2,5}$ Measured minimum stable cluster sizes for trivalent lanthanides ${ }^{5}$ as well as divalent metal ions ${ }^{6}$ are linearly related to their solution phase hydrolysis constants. For nine divalent metal ions where the minimum stable cluster size ranges from zero to 26 water molecules, the hydrolysis constant spans 10 orders of magnitude. ${ }^{6}$ IRPD spectroscopy indicates that several of these ions are in their divalent form in clusters at and above their critical size. For example, the minimum stable cluster size for $\mathrm{Mn}^{2+}$ is five whereas that for $\mathrm{Cu}^{2+}$ is eight. ${ }^{6}$ IRPD spectra of both ions as well as computations are consistent with the divalent form in these respective clusters. ${ }^{7,8}$ This indicates that the charge separation reaction that occurs when there are fewer water molecules than the critical size does not likely occur in the larger droplets. The stability of trivalent ions in aqueous nanodrops is also supported by gas phase electrochemistry experiments in which $\mathrm{Eu}^{3+}$ in clusters containing between 55 and 140 water molecules is directly reduced to form the $\mathrm{Eu}^{2+}\left(\mathrm{H}_{2} \mathrm{O}\right)_{n}$ products. ${ }^{9}$ These results suggest that the nanodrops in these experiments, where $n \geq 50$, should be sufficiently large to stabilize $\mathrm{La}^{3+}$.

MD Simulations. Molecular dynamics simulations were performed using the MacroModel dynamics software (Shrödinger, Inc., Portland, OR) to generate low-energy structures of $\mathrm{Mo}^{3+}\left(\mathrm{H}_{2} \mathrm{O}\right)_{550} . \mathrm{Mo}^{3+}$ is the largest trivalent metal ion for which there are available parameters for the OPLS 2005 force field implemented in this software, and was therefore used as an analog for $\mathrm{La}^{3+}$. The crystallographic ionic radius of $\mathrm{Mo}^{3+}$ is $70 \%$ of $\mathrm{La}^{3+}(83$ and $117 \mathrm{pm}$, respectively) ${ }^{10}$ and this difference in size may lead to some discrepancies in how the ions affect 
the H-bonding network of water, particularly in the primary hydration shell. Previous results from our laboratory indicate that the free $\mathrm{O}-\mathrm{H}$ stretches of water molecules located remotely from the ion on the surface of aqueous nanodrops are affected primarily by an ion's charge state with ion size having much smaller effect, ${ }^{11}$ and we expect that charge state is the most important parameter for solvent patterning at these large cluster sizes. The excluded volume of either ion in a cluster with 550 water molecules is less than $0.1 \%$ of the total nanodrop volume. After an initial geometry relaxation of $\mathrm{Mo}^{3+}\left(\mathrm{H}_{2} \mathrm{O}\right)_{550}$ using molecular mechanics, the system was allowed to equilibrate for $10 \mathrm{~ns}$ using canonical ensemble stochastic dynamics at $133 \mathrm{~K}$. Integration time steps of $1.5 \mathrm{fs}$ were used and the SHAKE algorithim was applied to all hydrogen atoms. From the final equilibrated geometry, a dynamics trajectory of $60 \mathrm{~ns}$ was run during which a structure was saved every 60 picoseconds generating 1000 structures for $\mathrm{Mo}^{3+}\left(\mathrm{H}_{2} \mathrm{O}\right)_{550}$. The position of $\mathrm{La}^{3+}$ within the cluster was not restrained, and the ion was internally solvated near the center of the droplet in the identified structures. An in-house routine written in MATLAB (The Mathworks Inc., Natick, MA) was used to calculate the angle $\theta$ between the dipole vector of each water molecule located at a distance $d$ from the metal ion and the vector defined by the metaloxygen displacement for all the identified structures. The resulting set of data $(d, \theta)$ was binned in $0.5 \AA$ increments, and the average angle $<\theta>$ in each bin was calculated. The cutoff distance for the exponential fit to the $\langle\theta>$ data in Figure 3 was determined from distance measurements performed on 20 structures equally spaced in time throughout the trajectory that indicate surface water molecules are located $15.5 \pm 1.6 \AA$ from the ion. Taking into account shell thicknesses of 2-3 Å (Figure 4), a distance cutoff of $13.5 \AA$ A was chosen. An additional 10 ns trajectory from a different starting geometry yielded a decay constant $\tau$ of $3.17 \AA$ indicating that the starting 
geometry does not have a significant impact on the distance of ion-water ordering in these

simulations.

\section{References:}

1. Bush, M. F.; O'Brien, J. T.; Prell, J. S.; Saykally, R. J.; Williams, E. R. J. Am. Chem. Soc. 2007, 129, 1612.

2. Bush, M. F.; Saykally, R. J.; Williams, E. R. Int. J. Mass. Spectrom. 2006, 253, 256.

3. Wong, R. L.; Paech, K.; Williams, E. R. Int. J. Mass. Spectrom. 2004, 232, 59.

4. Prell, J. S.; O'Brien, J. T.; Williams, E. R. J. Am. Soc. Mass. Spectr. 2010, 21, 800.

5. Bush, M. F.; Saykally, R. J.; Williams, E. R. J. Am. Chem. Soc. 2008, 130, 9122.

6. Chen, X. J.; Stace, A. J. Chem. Commun. 2012, 48, 10292.

7. O'Brien, J. T.; Williams, E. R. J. Phys. Chem. A 2008, 112, 5893.

8. O'Brien, J. T.; Williams, E. R. J. Phys. Chem. A 2011, 115, 14612.

9. Donald, W. A.; Leib, R. D.; Demireva, M.; O'Brien, J. T.; Prell, J. S.; Williams, E. R. J. Am. Chem. Soc. 2009, 131, 13328.

10. Shannon, R. D. Acta. Crystallogr. A 1976, 32, 751.

11. Prell, J. S.; O’Brien, J. T.; Williams, E. R. J. Am. Chem. Soc. 2011, 133, 4810.

\section{XYZ Coordinates of Structure for $\mathrm{Mo}^{3+}\left(\mathrm{H}_{2} \mathrm{O}\right)_{550}$ in Figure 4}

Note: $42=\mathrm{Mo}, 8=\mathrm{O}, 1=\mathrm{H}$

$\begin{array}{lccc}42 & 0.578400000 & -0.149500000 & 0.004500000 \\ 8 & -5.750700000 & -7.635700000 & -11.380100000 \\ 1 & -6.422700000 & -8.315100000 & -11.725200000 \\ 1 & -5.377700000 & -7.981700000 & -10.516000000 \\ 8 & 5.892600000 & 0.572800000 & -5.653300000 \\ 1 & 5.505100000 & 0.397400000 & -6.571000000 \\ 1 & 5.557300000 & 1.477800000 & -5.347500000 \\ 8 & -7.586800000 & 2.047600000 & -4.142100000 \\ 1 & -7.540800000 & 1.168700000 & -4.641400000 \\ 1 & -6.897800000 & 2.024800000 & -3.400600000 \\ 8 & -9.686400000 & 3.741500000 & 11.588900000 \\ 1 & -10.422700000 & 4.010700000 & 10.952400000 \\ 1 & -9.686800000 & 2.735200000 & 11.676000000 \\ 8 & -6.851600000 & 6.714800000 & 0.729000000 \\ 1 & -7.324500000 & 6.191100000 & 0.003500000 \\ 1 & -7.059000000 & 6.315800000 & 1.634400000 \\ 8 & -8.402700000 & -6.505100000 & 3.743600000 \\ 1 & -7.891400000 & -6.492700000 & 4.616300000 \\ 1 & -9.376500000 & -6.653800000 & 3.972800000 \\ 8 & 3.453100000 & 10.100900000 & -5.398600000 \\ 1 & 4.043400000 & 10.601000000 & -6.050600000 \\ 1 & 2.881400000 & 9.449000000 & -5.923400000\end{array}$




$\begin{array}{cccc}8 & 2.436700000 & 4.529500000 & -13.797500000 \\ 1 & 3.397500000 & 4.277400000 & -13.609400000 \\ 1 & 2.076300000 & 3.928600000 & -14.525200000 \\ 8 & -4.243400000 & -5.457200000 & 10.341100000 \\ 1 & -3.693300000 & -6.212400000 & 10.730300000 \\ 1 & -5.196800000 & -5.552300000 & 10.668600000 \\ 8 & 4.846700000 & 3.727300000 & -12.899100000 \\ 1 & 5.821700000 & 3.816700000 & -13.154000000 \\ 1 & 4.726500000 & 4.146800000 & -11.985500000 \\ 8 & -3.229100000 & 2.003900000 & 4.929400000 \\ 1 & -3.435400000 & 1.016000000 & 5.009600000 \\ 1 & -2.472200000 & 2.131100000 & 4.271200000 \\ 8 & 2.474200000 & -11.395000000 & 5.561000000 \\ 1 & 1.524500000 & -11.179500000 & 5.837200000 \\ 1 & 2.694200000 & -10.948800000 & 4.679700000 \\ 8 & -3.911700000 & -1.877000000 & -2.164600000 \\ 1 & -3.618900000 & -0.977600000 & -2.525200000 \\ 1 & -3.720900000 & -2.594600000 & -2.851300000 \\ 8 & 12.924200000 & 6.656500000 & 1.421500000 \\ 1 & 12.541900000 & 5.921300000 & 0.838800000 \\ 1 & 13.875100000 & 6.822700000 & 1.147800000 \\ 8 & 9.329600000 & 10.496900000 & -2.371700000 \\ 1 & 9.800400000 & 11.156700000 & -1.769000000 \\ 1 & 8.337200000 & 10.572200000 & -2.186200000 \\ 8 & -4.526800000 & -11.228800000 & 1.755300000 \\ 1 & -4.927900000 & -10.759200000 & 2.557900000 \\ 1 & -4.253900000 & -12.157600000 & 2.050500000 \\ 8 & 12.936700000 & -1.343300000 & -6.122600000 \\ 1 & 12.309400000 & -2.099700000 & -5.882400000 \\ 1 & 13.178400000 & -1.445900000 & -7.097900000 \\ 8 & 2.249000000 & -6.003200000 & -1.357800000 \\ 1 & 3.024900000 & -5.379800000 & -1.534000000 \\ 1 & 1.740500000 & -6.192700000 & -2.211800000 \\ 8 & 6.132900000 & -1.425600000 & 11.032900000 \\ 1 & 6.922100000 & -1.692500000 & 11.606500000 \\ 1 & 5.471400000 & -2.190200000 & 10.974900000 \\ 8 & -10.811800000 & -5.063700000 & -4.922800000 \\ 1 & -11.140900000 & -4.395600000 & -4.242800000 \\ 1 & -10.642800000 & -4.579600000 & -5.792500000 \\ 8 & -9.549200000 & 2.545700000 & 2.556900000 \\ 1 & -10.221300000 & 1.843600000 & 2.269200000 \\ 1 & -8.671400000 & 2.061300000 & 2.695700000 \\ 8 & -5.123300000 & -5.258300000 & -2.365600000 \\ 1 & -5.989200000 & -5.249500000 & -2.892000000 \\ 8 & -4.501500000 & -4.578100000 & -2.782400000 \\ & 4.751400000 & -5.918700000 & 3.556700000\end{array}$




$\begin{array}{cccc}1 & 5.015300000 & -5.205600000 & 2.889100000 \\ 1 & 4.625200000 & -6.776100000 & 3.033500000 \\ 8 & 9.246800000 & -0.603000000 & -8.225600000 \\ 1 & 8.524500000 & -0.732900000 & -8.923300000 \\ 1 & 8.808600000 & -0.596000000 & -7.312500000 \\ 8 & 11.467400000 & 1.073600000 & 9.548900000 \\ 1 & 11.746400000 & 1.213100000 & 8.586800000 \\ 1 & 12.022000000 & 0.324300000 & 9.939100000 \\ 8 & -0.303500000 & -14.723200000 & 5.105500000 \\ 1 & -0.747600000 & -15.043900000 & 5.962300000 \\ 1 & 0.673200000 & -14.575200000 & 5.279000000 \\ 8 & -2.347100000 & -7.084500000 & -9.349400000 \\ 1 & -3.149800000 & -7.686100000 & -9.344200000 \\ 1 & -2.347600000 & -6.497600000 & -8.522700000 \\ 8 & -3.801500000 & 0.912800000 & 8.676400000 \\ 1 & -3.721600000 & 1.865600000 & 9.003600000 \\ 1 & -4.781000000 & 0.656800000 & 8.644700000 \\ 8 & 3.721600000 & -5.096800000 & -10.477900000 \\ 1 & 2.847000000 & -5.375400000 & -10.899800000 \\ 1 & 3.733000000 & -5.435200000 & -9.524500000 \\ 8 & -0.157500000 & 6.823100000 & 5.593900000 \\ 1 & 0.521100000 & 6.893100000 & 6.340800000 \\ 1 & -0.021400000 & 7.571900000 & 4.920800000 \\ 8 & -13.630200000 & 4.501400000 & 1.357500000 \\ 1 & -13.931300000 & 5.416800000 & 1.059700000 \\ 1 & -12.896800000 & 4.645800000 & 2.036200000 \\ 8 & -1.065100000 & 3.782500000 & -15.024600000 \\ 1 & -0.824000000 & 4.709000000 & -14.707500000 \\ 1 & -2.001300000 & 3.573600000 & -14.704300000 \\ 8 & 9.065700000 & -6.074800000 & -6.256900000 \\ 1 & 9.049400000 & -5.062100000 & -6.246400000 \\ 1 & 10.027400000 & -6.388800000 & -6.266600000 \\ 8 & 7.898000000 & 2.369600000 & 2.655500000 \\ 1 & 7.814300000 & 1.380800000 & 2.445600000 \\ 1 & 7.899800000 & 2.464700000 & 3.662900000 \\ 8 & -0.338100000 & 3.318800000 & 7.425300000 \\ 1 & -0.415900000 & 4.127600000 & 8.029500000 \\ 1 & -0.404000000 & 3.643000000 & 6.468400000 \\ 8 & 1.822700000 & -0.232600000 & -1.795100000 \\ 1 & 2.083600000 & 0.544700000 & -2.400700000 \\ 1 & 2.320500000 & -1.059900000 & -2.116200000 \\ 8 & 11.989600000 & -6.438700000 & -5.939500000 \\ 1 & 12.365900000 & -6.404000000 & -4.995800000 \\ 8 & 12.584200000 & -7.062200000 & -6.452900000 \\ 1 & 10.922100000 & 3.020800000 & -6.633600000 \\ & 10.081200000 & 3.524900000 & -6.382700000\end{array}$




$\begin{array}{lccc}1 & 10.669400000 & 2.375400000 & -7.371300000 \\ 8 & 5.745800000 & -5.898400000 & -3.916300000 \\ 1 & 5.100100000 & -6.678400000 & -3.920800000 \\ 1 & 6.669300000 & -6.310900000 & -3.952000000 \\ 8 & -7.429100000 & -0.374100000 & -5.553500000 \\ 1 & -8.141000000 & -0.689000000 & -4.905900000 \\ 1 & -7.814200000 & -0.441400000 & -6.487800000 \\ 8 & -1.258900000 & 1.066000000 & -0.129300000 \\ 1 & -1.077400000 & 2.066000000 & -0.221700000 \\ 1 & -2.241700000 & 0.994900000 & 0.132000000 \\ 8 & -0.927500000 & -0.186000000 & -6.787700000 \\ 1 & -1.837800000 & 0.131300000 & -7.095900000 \\ 1 & -0.613800000 & -0.959200000 & -7.361400000 \\ 8 & 4.050100000 & 4.123400000 & -1.481400000 \\ 1 & 4.427400000 & 4.686200000 & -0.732100000 \\ 1 & 3.046500000 & 4.249500000 & -1.510600000 \\ 8 & -8.273600000 & -8.832900000 & -6.091800000 \\ 1 & -9.079000000 & -9.069700000 & -6.653500000 \\ 1 & -8.612000000 & -8.245000000 & -5.338600000 \\ 8 & 0.988000000 & -4.085300000 & 3.515000000 \\ 1 & -0.018200000 & -3.968100000 & 3.456300000 \\ 1 & 1.165800000 & -4.669900000 & 4.322600000 \\ 8 & 1.880500000 & 6.934800000 & 7.866600000 \\ 1 & 2.344600000 & 7.762500000 & 7.517100000 \\ 1 & 2.538000000 & 6.167600000 & 7.932400000 \\ 8 & 0.427000000 & 0.894900000 & 2.077000000 \\ 1 & -0.432800000 & 1.232300000 & 2.511000000 \\ 1 & 1.158800000 & 1.114000000 & 2.747000000 \\ 8 & 0.065900000 & -14.738400000 & -2.476300000 \\ 1 & 1.005300000 & -14.555100000 & -2.809200000 \\ 1 & -0.083500000 & -15.728500000 & -2.417600000 \\ 8 & 5.367000000 & -3.904700000 & 1.830000000 \\ 1 & 4.409600000 & -3.921900000 & 1.499100000 \\ 1 & 5.948600000 & -3.823500000 & 1.005300000 \\ 8 & 8.219200000 & -3.517000000 & -6.694100000 \\ 1 & 8.318600000 & -2.572100000 & -6.345600000 \\ 1 & 8.067200000 & -3.521100000 & -7.695600000 \\ 8 & -10.888500000 & -1.703500000 & 1.085600000 \\ 1 & -10.241000000 & -2.342100000 & 0.644500000 \\ 1 & -11.815600000 & -2.092400000 & 0.968000000 \\ 8 & -10.728500000 & 0.115200000 & -2.033300000 \\ 1 & -11.055500000 & 1.066800000 & -2.148100000 \\ 1 & -11.442000000 & -0.396200000 & -1.531200000 \\ 1 & 5.482400000 & 3.275200000 & 1.686600000 \\ 1 & 6.430700000 & 3.080800000 & 1.986300000 \\ & 5.431100000 & 4.178400000 & 1.231900000\end{array}$




$\begin{array}{cccc}8 & 4.083600000 & 4.587700000 & -10.470100000 \\ 1 & 3.145200000 & 4.214500000 & -10.532400000 \\ 1 & 4.585500000 & 4.174900000 & -9.694300000 \\ 8 & 1.638500000 & 0.909700000 & -6.341500000 \\ 1 & 0.629700000 & 0.838300000 & -6.393600000 \\ 1 & 2.052900000 & 0.056100000 & -6.694800000 \\ 8 & -6.700300000 & 3.901100000 & -6.031000000 \\ 1 & -7.321500000 & 4.697900000 & -6.085700000 \\ 1 & -7.065800000 & 3.296000000 & -5.306000000 \\ 8 & 2.266100000 & 1.630900000 & 3.851200000 \\ 1 & 3.213500000 & 1.273600000 & 3.828500000 \\ 1 & 2.012400000 & 1.727200000 & 4.826500000 \\ 8 & 14.612200000 & -5.240400000 & 0.792400000 \\ 1 & 14.078200000 & -5.210400000 & 1.650300000 \\ 1 & 14.596900000 & -6.200400000 & 0.480700000 \\ 8 & 12.137800000 & 7.323000000 & 5.832700000 \\ 1 & 11.144300000 & 7.538600000 & 5.828800000 \\ 1 & 12.659700000 & 8.175300000 & 5.749200000 \\ 8 & 4.274100000 & 7.262400000 & -11.151400000 \\ 1 & 4.281000000 & 6.285000000 & -10.888500000 \\ 1 & 5.222400000 & 7.592600000 & -11.257900000 \\ 8 & 8.883400000 & -0.796200000 & 7.913600000 \\ 1 & 9.015800000 & -0.296400000 & 8.784400000 \\ 1 & 9.255800000 & -0.218100000 & 7.171000000 \\ 8 & -2.766300000 & -11.382300000 & -4.242400000 \\ 1 & -2.693300000 & -10.577600000 & -4.849400000 \\ 1 & -3.736000000 & -11.655800000 & -4.151100000 \\ 8 & -9.026700000 & -11.042600000 & 0.747100000 \\ 1 & -9.604100000 & -11.772900000 & 0.373800000 \\ 1 & -8.107700000 & -11.158300000 & 0.336500000 \\ 8 & 2.102400000 & -14.772600000 & 2.721100000 \\ 1 & 2.311500000 & -14.674800000 & 3.706000000 \\ 1 & 1.833300000 & -15.730500000 & 2.545600000 \\ 8 & 11.458500000 & -3.779700000 & -6.263200000 \\ 1 & 11.155100000 & -3.518200000 & -7.191300000 \\ 1 & 11.756400000 & -4.746100000 & -6.223700000 \\ 8 & 1.201800000 & 0.907400000 & -12.121400000 \\ 1 & 1.590100000 & 0.676000000 & -13.026300000 \\ 1 & 1.559900000 & 1.822900000 & -11.876200000 \\ 8 & -11.430200000 & 4.386300000 & 5.437800000 \\ 1 & -10.772600000 & 3.619700000 & 5.370400000 \\ 1 & -11.643900000 & 4.690700000 & 4.496400000 \\ 8 & 5.444100000 & 7.947200000 & -2.053500000 \\ 1 & 6.201700000 & 7.740200000 & -1.418300000 \\ 8 & 5.729700000 & 7.635100000 & -2.972900000 \\ & 0.975600000 & 5.461000000 & 1.343200000\end{array}$




\begin{tabular}{rrrr}
1 & 1.559600000 & 4.651000000 & 1.508000000 \\
1 & 1.556100000 & 6.286700000 & 1.355400000 \\
8 & 1.343200000 & -4.552700000 & -5.989300000 \\
1 & 2.324100000 & -4.372600000 & -6.166100000 \\
1 & 0.816800000 & -4.592300000 & -6.853800000 \\
8 & -3.250700000 & 2.855600000 & -13.213400000 \\
1 & -4.108700000 & 2.744400000 & -12.684500000 \\
1 & -2.829900000 & 3.727100000 & -12.920700000 \\
8 & -9.202900000 & -1.337200000 & -3.747200000 \\
1 & -9.705000000 & -0.797600000 & -3.053000000 \\
1 & -9.839200000 & -1.738900000 & -4.424200000 \\
8 & -0.953300000 & 3.158400000 & -6.406500000 \\
1 & -1.244800000 & 4.095900000 & -6.653600000 \\
1 & -1.101900000 & 2.552400000 & -7.203600000 \\
8 & 6.364200000 & -1.722500000 & 8.267100000 \\
1 & 6.075700000 & -1.610300000 & 9.230000000 \\
1 & 7.302600000 & -1.359600000 & 8.158000000 \\
8 & -4.407900000 & 9.068200000 & 6.811600000 \\
1 & -3.854600000 & 8.257700000 & 6.570700000 \\
1 & -5.287800000 & 8.747800000 & 7.197300000 \\
8 & 6.364900000 & 7.057800000 & -4.373400000 \\
1 & 6.331900000 & 7.803400000 & -5.056700000 \\
1 & 5.628000000 & 6.391800000 & -4.571200000 \\
8 & 7.919300000 & -3.417200000 & -9.423300000 \\
1 & 7.357800000 & -4.085700000 & -9.936100000 \\
1 & 7.876200000 & -2.512400000 & -9.871000000 \\
8 & -2.551300000 & 5.296000000 & -1.024700000 \\
1 & -1.661800000 & 5.734200000 & -1.219000000 \\
1 & -3.158500000 & 5.983400000 & -0.590000000 \\
8 & -9.320300000 & 8.752400000 & -3.445000000 \\
1 & -8.418700000 & 8.441700000 & -3.785000000 \\
1 & -10.073700000 & 8.200300000 & -3.830200000 \\
8 & 12.128300000 & 1.209500000 & 3.100000000 \\
1 & 12.587900000 & 1.853900000 & 2.470100000 \\
1 & 11.348200000 & 0.789200000 & 2.611200000 \\
8 & -2.945900000 & -2.928600000 & 10.307400000 \\
1 & -3.520200000 & -2.212700000 & 10.736200000 \\
1 & -3.268800000 & -3.851300000 & 10.569900000 \\
8 & 0.304300000 & -13.882800000 & 8.282400000 \\
1 & -0.193100000 & -13.045000000 & 8.520000000 \\
1 & -0.387100000 & -14.554600000 & 7.970400000 \\
8 & 3.831500000 & 4.629400000 & 7.761700000 \\
1 & 3.589200000 & 4.142600000 & 8.616600000 \\
1 & 4.832700000 & 4.784900000 & 7.750500000 \\
& -4.489600000 & 7.886300000 & -5.544600000 \\
\hline & &
\end{tabular}




$\begin{array}{cccc}1 & -4.140300000 & 8.062500000 & -4.613200000 \\ 8 & -9.695300000 & 8.007000000 & 10.319000000 \\ 1 & -10.439000000 & 7.804000000 & 9.676900000 \\ 1 & -10.114000000 & 8.078600000 & 11.238100000 \\ 8 & 1.206500000 & -2.359000000 & -0.133200000 \\ 1 & 0.600600000 & -2.939800000 & -0.706000000 \\ 1 & 1.865400000 & -2.960300000 & 0.354400000 \\ 8 & 10.452300000 & 10.755800000 & 1.604400000 \\ 1 & 10.562400000 & 9.757800000 & 1.474100000 \\ 1 & 9.760400000 & 10.947800000 & 2.304800000 \\ 8 & 6.994400000 & 2.383000000 & 9.625300000 \\ 1 & 7.440200000 & 3.236200000 & 9.926800000 \\ 1 & 7.618200000 & 1.634600000 & 9.894700000 \\ 8 & -6.960000000 & 1.354300000 & -9.324500000 \\ 1 & -6.351500000 & 1.897300000 & -8.724200000 \\ 1 & -6.436200000 & 0.857700000 & -10.034400000 \\ 8 & 4.344300000 & 5.394200000 & -5.064800000 \\ 1 & 4.309700000 & 5.861200000 & -5.962700000 \\ 1 & 3.384900000 & 5.245800000 & -4.777500000 \\ 8 & -3.894500000 & -7.392600000 & -5.592200000 \\ 1 & -4.778600000 & -7.520600000 & -6.066700000 \\ 1 & -3.974800000 & -7.538700000 & -4.593000000 \\ 8 & 2.808800000 & -4.640100000 & -13.696200000 \\ 1 & 3.581900000 & -5.044300000 & -14.188700000 \\ 1 & 2.632500000 & -3.708400000 & -14.047500000 \\ 8 & 5.021900000 & -1.409100000 & 5.913100000 \\ 1 & 4.022400000 & -1.326800000 & 6.049200000 \\ 1 & 5.459500000 & -1.490200000 & 6.819700000 \\ 8 & -1.382400000 & -8.341000000 & 8.322100000 \\ 1 & -0.545300000 & -8.366400000 & 7.754500000 \\ 1 & -1.119900000 & -7.892700000 & 9.188900000 \\ 8 & -11.037600000 & 10.195400000 & 6.083200000 \\ 1 & -10.293400000 & 9.557000000 & 6.347600000 \\ 1 & -10.627300000 & 11.063500000 & 5.767100000 \\ 8 & -2.648100000 & -5.433600000 & -7.161800000 \\ 1 & -2.972000000 & -6.166200000 & -6.541200000 \\ 1 & -3.311500000 & -4.669900000 & -7.127000000 \\ 8 & 5.791000000 & 16.622300000 & 0.780300000 \\ 1 & 5.848300000 & 17.615800000 & 0.653100000 \\ 1 & 4.951100000 & 16.408700000 & 1.302100000 \\ 8 & -0.354900000 & 6.576300000 & -1.725200000 \\ 1 & -0.680800000 & 7.001300000 & -2.583500000 \\ 1 & 0.379300000 & 7.158200000 & -1.344400000 \\ 8 & 8.447800000 & -4.326700000 & -2.070800000 \\ 1 & 8.756800000 & -5.255300000 & -1.814800000 \\ 1 & 9.038000000 & -3.957900000 & -2.804800000\end{array}$




$\begin{array}{cccc}8 & 4.872900000 & 3.156500000 & -8.211400000 \\ 1 & 5.791500000 & 3.415500000 & -7.875500000 \\ 1 & 4.808900000 & 2.148000000 & -8.261400000 \\ 8 & 2.824300000 & 9.983600000 & 2.894400000 \\ 1 & 3.512100000 & 10.171200000 & 3.603200000 \\ 1 & 2.960500000 & 10.641100000 & 2.141400000 \\ 8 & -6.041400000 & -13.817300000 & -1.845600000 \\ 1 & -5.204400000 & -13.936800000 & -1.285700000 \\ 1 & -6.391700000 & -14.736900000 & -2.041000000 \\ 8 & -4.954300000 & 6.708400000 & 3.955000000 \\ 1 & -4.275700000 & 6.499300000 & 4.677100000 \\ 1 & -4.970200000 & 7.711400000 & 3.830000000 \\ 8 & -7.717600000 & -11.555900000 & 6.332300000 \\ 1 & -8.040700000 & -10.660000000 & 6.662300000 \\ 1 & -7.959600000 & -11.623100000 & 5.355300000 \\ 8 & -13.215400000 & 2.221100000 & -0.086300000 \\ 1 & -13.977700000 & 1.566500000 & 0.022000000 \\ 1 & -13.494200000 & 3.057400000 & 0.417900000 \\ 8 & 9.436400000 & -7.527200000 & 7.043900000 \\ 1 & 9.411100000 & -7.696700000 & 6.047300000 \\ 1 & 10.395600000 & -7.540600000 & 7.363200000 \\ 8 & -13.304800000 & 3.778100000 & 7.186800000 \\ 1 & -14.188100000 & 4.121600000 & 6.837000000 \\ 1 & -12.598800000 & 3.991900000 & 6.495500000 \\ 8 & 2.941200000 & 2.923200000 & 9.992800000 \\ 1 & 3.374800000 & 2.349600000 & 10.706000000 \\ 1 & 1.963700000 & 3.059500000 & 10.207900000 \\ 8 & -11.575600000 & -4.859700000 & -1.083700000 \\ 1 & -11.763500000 & -4.211600000 & -1.835700000 \\ 1 & -12.436000000 & -5.045600000 & -0.586700000 \\ 8 & -2.912600000 & -2.588800000 & 0.338300000 \\ 1 & -3.151100000 & -2.294500000 & -0.600900000 \\ 1 & -3.295400000 & -3.510200000 & 0.501300000 \\ 8 & -5.714400000 & 8.759100000 & -9.807800000 \\ 1 & -6.117100000 & 9.592300000 & -10.190700000 \\ 1 & -5.059500000 & 9.011300000 & -9.077700000 \\ 8 & -5.684700000 & -3.354400000 & 2.413000000 \\ 1 & -6.646700000 & -3.666600000 & 2.421400000 \\ 1 & -5.166400000 & -3.962800000 & 1.795500000 \\ 8 & -15.110400000 & 0.257100000 & 0.135100000 \\ 1 & -16.019600000 & 0.533800000 & -0.184900000 \\ 1 & -15.183800000 & -0.199100000 & 1.039600000 \\ 8 & -6.258300000 & 5.486700000 & -2.842600000 \\ 1 & -6.956100000 & 5.224200000 & -2.159500000 \\ 1 & -6.571300000 & 6.347200000 & -3.266700000 \\ 8 & 1.271300000 & 2.587900000 & -15.616500000\end{array}$




\begin{tabular}{cccc}
1 & 1.352400000 & 2.418300000 & -16.601900000 \\
1 & 0.365300000 & 3.016200000 & -15.469600000 \\
8 & 8.954000000 & 6.295700000 & -3.371100000 \\
1 & 8.086900000 & 6.662400000 & -3.743300000 \\
1 & 9.283900000 & 6.941200000 & -2.664800000 \\
8 & 4.357200000 & -3.468500000 & 10.911800000 \\
1 & 4.105800000 & -4.440200000 & 10.754300000 \\
1 & 3.679900000 & -3.089700000 & 11.561900000 \\
8 & -2.093800000 & 5.150900000 & -11.968900000 \\
1 & -1.535200000 & 5.602700000 & -12.680500000 \\
1 & -2.765900000 & 5.839200000 & -11.656100000 \\
8 & -4.441000000 & -3.376400000 & -6.779400000 \\
1 & -4.238000000 & -3.085200000 & -7.729000000 \\
1 & -4.579500000 & -2.543400000 & -6.221300000 \\
8 & -1.002900000 & -2.712500000 & -14.323400000 \\
1 & -0.705300000 & -2.736800000 & -15.286700000 \\
1 & -1.906400000 & -2.260000000 & -14.265900000 \\
8 & 11.576900000 & -2.280400000 & 0.427600000 \\
1 & 11.790900000 & -2.400000000 & -0.553000000 \\
1 & 12.424800000 & -2.069900000 & 0.937300000 \\
8 & -5.730600000 & 1.646800000 & -2.087800000 \\
1 & -6.392000000 & 1.086800000 & -1.566900000 \\
1 & -5.385100000 & 2.377400000 & -1.477500000 \\
8 & -2.328800000 & 5.980600000 & 10.741100000 \\
1 & -2.202100000 & 5.931000000 & 11.734300000 \\
1 & -3.135100000 & 6.558700000 & 10.522600000 \\
8 & 6.704000000 & 10.889500000 & -1.632100000 \\
1 & 6.244700000 & 10.368400000 & -0.895500000 \\
1 & 6.004500000 & 11.027300000 & -2.350400000 \\
8 & -3.052100000 & -9.375800000 & 0.473000000 \\
1 & -2.730500000 & -9.703200000 & -0.429100000 \\
1 & -3.657400000 & -10.096500000 & 0.846700000 \\
8 & -6.261600000 & -7.282700000 & -6.925400000 \\
1 & -6.995300000 & -7.912600000 & -6.639500000 \\
1 & -6.636800000 & -6.344500000 & -6.910500000 \\
8 & 12.796200000 & -4.145000000 & 7.215000000 \\
1 & 12.819300000 & -3.573100000 & 6.383800000 \\
1 & 11.921600000 & -3.982600000 & 7.692400000 \\
8 & -6.963400000 & -4.632600000 & -6.615000000 \\
1 & -7.518100000 & -4.112600000 & -7.282200000 \\
1 & -6.047700000 & -4.205300000 & -6.561900000 \\
8 & 4.975400000 & 0.703600000 & 3.903300000 \\
1 & 4.897100000 & -0.060700000 & 4.561700000 \\
1 & 5.672200000 & 0.474900000 & 3.206700000 \\
& -3.344600000 & 4.144500000 & 6.599700000 \\
\hline & -3.353100000 & 3.815600000 & 7.555500000
\end{tabular}




$\begin{array}{cccc}1 & -3.251500000 & 3.314500000 & 6.029300000 \\ 8 & 7.958000000 & -7.296300000 & -3.955800000 \\ 1 & 8.548800000 & -6.929800000 & -4.691800000 \\ 1 & 8.444100000 & -7.206900000 & -3.070700000 \\ 8 & -3.531100000 & -4.838900000 & 7.753500000 \\ 1 & -4.217900000 & -4.156800000 & 7.463100000 \\ 1 & -3.758900000 & -5.129500000 & 8.696500000 \\ 8 & 8.954100000 & 0.539300000 & 10.302300000 \\ 1 & 9.906100000 & 0.865500000 & 10.209600000 \\ 1 & 8.908400000 & -0.099100000 & 11.085600000 \\ 8 & 11.716500000 & -6.614900000 & -0.659600000 \\ 1 & 11.558800000 & -5.941300000 & 0.078900000 \\ 1 & 12.521500000 & -7.171100000 & -0.402400000 \\ 8 & -4.114400000 & -13.864100000 & 2.487000000 \\ 1 & -3.145700000 & -13.756800000 & 2.758300000 \\ 1 & -4.535000000 & -14.496400000 & 3.154100000 \\ 8 & -10.993400000 & 6.865700000 & 6.373900000 \\ 1 & -10.154800000 & 7.320300000 & 6.037000000 \\ 1 & -10.980500000 & 5.900400000 & 6.069700000 \\ 8 & -3.499400000 & 0.799800000 & -7.299700000 \\ 1 & -4.138900000 & 1.568400000 & -7.454900000 \\ 1 & -3.869100000 & 0.255000000 & -6.530500000 \\ 8 & -7.933000000 & 13.158000000 & -1.469600000 \\ 1 & -8.269000000 & 14.017300000 & -1.884700000 \\ 1 & -7.901800000 & 12.404000000 & -2.141500000 \\ 8 & -8.887200000 & 8.540600000 & 5.805600000 \\ 1 & -8.516800000 & 9.079400000 & 5.038200000 \\ 1 & -8.088800000 & 8.240100000 & 6.347200000 \\ 8 & 4.943300000 & 11.037800000 & -7.464900000 \\ 1 & 4.416100000 & 11.254000000 & -8.298800000 \\ 1 & 5.304800000 & 11.914600000 & -7.112000000 \\ 8 & -0.429700000 & -11.055900000 & 11.148000000 \\ 1 & 0.346600000 & -10.449300000 & 10.914200000 \\ 1 & -0.147200000 & -11.709400000 & 11.854500000 \\ 8 & 7.042200000 & 9.117400000 & 2.116500000 \\ 1 & 7.548700000 & 9.866200000 & 2.568600000 \\ 1 & 6.624500000 & 8.558300000 & 2.850900000 \\ 8 & 10.239200000 & -3.329800000 & -3.753000000 \\ 1 & 11.051800000 & -3.159700000 & -3.173300000 \\ 1 & 10.560700000 & -3.558800000 & -4.684700000 \\ 8 & -1.812800000 & 7.567100000 & -8.806900000 \\ 1 & -0.933200000 & 7.523800000 & -9.304800000 \\ 1 & -1.820500000 & 8.354800000 & -8.171600000 \\ 8 & 1.422100000 & 8.266500000 & -0.826200000 \\ 1 & 0.731800000 & 9.005800000 & -0.870500000 \\ 1 & 2.061300000 & 8.358000000 & -1.607400000\end{array}$




\begin{tabular}{cccc}
8 & 3.848500000 & -3.616400000 & -6.651400000 \\
1 & 4.542300000 & -3.483000000 & -5.925500000 \\
1 & 3.992200000 & -4.526700000 & -7.070000000 \\
8 & 2.312700000 & 8.088400000 & -6.676000000 \\
1 & 2.962800000 & 7.438200000 & -7.100500000 \\
1 & 1.516000000 & 7.531400000 & -6.392200000 \\
8 & 12.700000000 & 1.361400000 & 6.916600000 \\
1 & 13.641100000 & 1.100300000 & 6.654300000 \\
1 & 12.385600000 & 2.120700000 & 6.326300000 \\
8 & 7.255500000 & -8.436400000 & 8.397900000 \\
1 & 8.065800000 & -7.989300000 & 7.989500000 \\
1 & 7.163700000 & -9.355300000 & 7.987000000 \\
8 & -5.803000000 & -9.985200000 & 3.815800000 \\
1 & -5.565600000 & -9.023400000 & 4.021200000 \\
1 & -6.796300000 & -10.143900000 & 3.932800000 \\
8 & 7.013000000 & 7.914100000 & -10.885400000 \\
1 & 7.426900000 & 8.582600000 & -11.507400000 \\
1 & 7.219800000 & 6.967400000 & -11.192600000 \\
8 & -0.810400000 & -1.416800000 & 1.293400000 \\
1 & -1.655200000 & -1.944400000 & 1.080700000 \\
1 & -0.652800000 & -1.524900000 & 2.293000000 \\
8 & -12.115300000 & -2.960400000 & -3.126000000 \\
1 & -12.447600000 & -2.349200000 & -2.393000000 \\
1 & -12.091900000 & -2.416200000 & -3.975600000 \\
8 & 11.930900000 & 3.188400000 & 5.010300000 \\
1 & 12.234600000 & 4.006900000 & 4.499800000 \\
1 & 11.804200000 & 2.439200000 & 4.342900000 \\
8 & 13.307900000 & -0.906700000 & 10.708400000 \\
1 & 13.677300000 & -1.573300000 & 10.039900000 \\
1 & 14.014300000 & -0.701600000 & 11.390300000 \\
8 & -5.400300000 & -7.358300000 & 13.235900000 \\
1 & -5.399200000 & -7.455300000 & 14.242700000 \\
1 & -4.461500000 & -7.245300000 & 12.876600000 \\
8 & -3.650900000 & -12.972000000 & 7.169800000 \\
1 & -4.594300000 & -13.181600000 & 7.464400000 \\
1 & -3.733100000 & -12.237900000 & 6.478800000 \\
8 & -3.907000000 & 4.426400000 & -3.197200000 \\
1 & -4.856800000 & 4.758000000 & -3.107500000 \\
1 & -3.414300000 & 4.661300000 & -2.343200000 \\
8 & -7.075100000 & 1.210600000 & 2.640900000 \\
1 & -6.387200000 & 1.902800000 & 2.915000000 \\
1 & -6.595400000 & 0.432700000 & 2.203500000 \\
8 & 6.517000000 & 5.509100000 & 7.869400000 \\
& 7.366100000 & 5.323800000 & 7.350400000 \\
1 & 6.716300000 & 5.321800000 & 8.844400000 \\
\hline & 4.151500000 & -8.072400000 & 12.089500000
\end{tabular}




$\begin{array}{cccc}1 & 4.102600000 & -8.124900000 & 13.089400000 \\ 1 & 5.090100000 & -8.360700000 & 11.831800000 \\ 8 & -5.173500000 & 3.316300000 & 0.114100000 \\ 1 & -4.673900000 & 3.976400000 & 0.696300000 \\ 1 & -6.148400000 & 3.358000000 & 0.379800000 \\ 8 & -9.545200000 & 5.332200000 & -9.127700000 \\ 1 & -9.289000000 & 4.516300000 & -9.667500000 \\ 1 & -8.722500000 & 5.913500000 & -9.050300000 \\ 8 & -11.855700000 & 7.353500000 & 8.812000000 \\ 1 & -11.594400000 & 7.068400000 & 7.875800000 \\ 1 & -12.605400000 & 8.029600000 & 8.753700000 \\ 8 & 10.447500000 & -1.768400000 & 4.070200000 \\ 1 & 9.911300000 & -2.624300000 & 4.152800000 \\ 1 & 10.163800000 & -1.276000000 & 3.233200000 \\ 8 & -9.097100000 & -1.804300000 & 12.346400000 \\ 1 & -8.296100000 & -2.335300000 & 12.026700000 \\ 1 & -9.297600000 & -2.052800000 & 13.297600000 \\ 8 & 1.843200000 & 1.596900000 & 6.606000000 \\ 1 & 2.523400000 & 1.305600000 & 7.294600000 \\ 1 & 1.139400000 & 2.136600000 & 7.095700000 \\ 8 & 13.690400000 & 2.579800000 & -7.235400000 \\ 1 & 12.699300000 & 2.647000000 & -7.036800000 \\ 1 & 14.073500000 & 3.501600000 & -7.333000000 \\ 8 & -7.987800000 & 11.241400000 & -3.407900000 \\ 1 & -8.346000000 & 10.307100000 & -3.247600000 \\ 1 & -8.260300000 & 11.532000000 & -4.328200000 \\ 8 & 0.300100000 & 3.078800000 & 10.659400000 \\ 1 & -0.372600000 & 2.462300000 & 10.225600000 \\ 1 & 0.009300000 & 4.034000000 & 10.482800000 \\ 8 & -10.677000000 & 4.953300000 & -5.797200000 \\ 1 & -11.235400000 & 4.779800000 & -6.622200000 \\ 1 & -10.578200000 & 4.063900000 & -5.322900000 \\ 8 & 6.144900000 & -3.426500000 & -4.982500000 \\ 1 & 6.974700000 & -3.436100000 & -5.560700000 \\ 1 & 6.132000000 & -4.273300000 & -4.424700000 \\ 8 & 1.489600000 & -6.664300000 & -4.010600000 \\ 1 & 1.443400000 & -5.967900000 & -4.743500000 \\ 1 & 0.881100000 & -7.434900000 & -4.256000000 \\ 8 & -14.122000000 & 3.321300000 & 3.741500000 \\ 1 & -14.241500000 & 3.671300000 & 2.809500000 \\ 1 & -14.544500000 & 3.965000000 & 4.400000000 \\ 8 & -8.263800000 & -4.024800000 & 2.535300000 \\ 1 & -8.708000000 & -4.083500000 & 1.627200000 \\ 8 & -8.292300000 & -4.958000000 & 2.922100000 \\ 1 & -3.121000000 & 5.921100000 & 6.196300000\end{array}$




$\begin{array}{cccc}1 & -1.888400000 & 6.968900000 & 5.857300000 \\ 8 & 4.520800000 & -4.584800000 & -1.963800000 \\ 1 & 5.140700000 & -4.262300000 & -1.234400000 \\ 1 & 5.066300000 & -5.086300000 & -2.650100000 \\ 8 & -8.285600000 & 5.201000000 & -1.154700000 \\ 1 & -8.275200000 & 4.304800000 & -0.683500000 \\ 1 & -9.248500000 & 5.412900000 & -1.380800000 \\ 8 & -11.090100000 & 9.565800000 & 3.373300000 \\ 1 & -11.235200000 & 10.463000000 & 2.933400000 \\ 1 & -11.166300000 & 9.635100000 & 4.378100000 \\ 8 & 14.081600000 & -0.487700000 & 4.042600000 \\ 1 & 14.621100000 & 0.049300000 & 4.707700000 \\ 1 & 13.321100000 & 0.084100000 & 3.700700000 \\ 8 & -5.237100000 & -11.238500000 & 9.858500000 \\ 1 & -5.761900000 & -11.980800000 & 9.416600000 \\ 1 & -4.779400000 & -10.679500000 & 9.149200000 \\ 8 & -1.530700000 & -13.739200000 & 2.946600000 \\ 1 & -0.851200000 & -13.564500000 & 2.217600000 \\ 1 & -1.074300000 & -14.283400000 & 3.667800000 \\ 8 & 10.340100000 & 3.715500000 & 9.485900000 \\ 1 & 10.717900000 & 2.800100000 & 9.689400000 \\ 1 & 10.831500000 & 4.123100000 & 8.713400000 \\ 8 & 12.806400000 & -5.956000000 & -3.220100000 \\ 1 & 12.285100000 & -6.158700000 & -2.378500000 \\ 1 & 13.505900000 & -5.251700000 & -3.029400000 \\ 8 & 6.402100000 & -8.693100000 & -6.129400000 \\ 1 & 6.677400000 & -7.967600000 & -6.785200000 \\ 1 & 7.000900000 & -8.654600000 & -5.326100000 \\ 8 & -11.557400000 & 2.870000000 & -2.090900000 \\ 1 & -12.263600000 & 2.524900000 & -1.450000000 \\ 1 & -11.511300000 & 3.875600000 & -1.983700000 \\ 8 & 12.565000000 & -2.791400000 & -2.345300000 \\ 1 & 13.290800000 & -3.488600000 & -2.237900000 \\ 1 & 13.040000000 & -1.958600000 & -2.661500000 \\ 8 & -12.313700000 & 0.195800000 & 10.707800000 \\ 1 & -13.066200000 & 0.476000000 & 11.320600000 \\ 1 & -12.446600000 & 0.561700000 & 9.773400000 \\ 8 & -4.194800000 & -7.901700000 & -2.948200000 \\ 1 & -4.784900000 & -8.657100000 & -2.623600000 \\ 1 & -4.508600000 & -7.034000000 & -2.533600000 \\ 8 & -12.592000000 & 1.245400000 & 8.242800000 \\ 1 & -11.600300000 & 1.416000000 & 8.143500000 \\ 1 & -13.033800000 & 2.141500000 & 8.084900000 \\ 8 & -2.163900000 & -9.565200000 & -5.981700000 \\ 1 & -2.712200000 & -10.070000000 & -6.664100000 \\ 1 & -2.559300000 & -8.637400000 & -5.897000000\end{array}$




$\begin{array}{cccc}8 & -7.743100000 & 9.247300000 & 1.064100000 \\ 1 & -7.334200000 & 8.324100000 & 1.028500000 \\ 1 & -8.668200000 & 9.251600000 & 0.660000000 \\ 8 & -9.917200000 & 9.322800000 & -0.837500000 \\ 1 & -9.795700000 & 9.156200000 & -1.826400000 \\ 1 & -10.654300000 & 8.715600000 & -0.504700000 \\ 8 & -0.720300000 & -1.142300000 & -1.567200000 \\ 1 & -0.812700000 & -0.762400000 & -2.507100000 \\ 1 & -0.973100000 & -2.127200000 & -1.563900000 \\ 8 & 2.321100000 & 3.468000000 & -6.952200000 \\ 1 & 1.894100000 & 2.561300000 & -6.806900000 \\ 1 & 3.276600000 & 3.341300000 & -7.257500000 \\ 8 & 9.067000000 & -6.870600000 & -1.440600000 \\ 1 & 10.016300000 & -6.944000000 & -1.096200000 \\ 1 & 8.431200000 & -7.170300000 & -0.712800000 \\ 8 & -5.991300000 & -8.767800000 & 10.829700000 \\ 1 & -5.769700000 & -9.755300000 & 10.802700000 \\ 1 & -5.648800000 & -8.406200000 & 11.709900000 \\ 8 & -11.628000000 & 7.438600000 & 0.108600000 \\ 1 & -11.153100000 & 7.128400000 & 0.948000000 \\ 1 & -12.622100000 & 7.377900000 & 0.282300000 \\ 8 & 11.237300000 & 4.880900000 & 7.148200000 \\ 1 & 11.474400000 & 4.204100000 & 6.434200000 \\ 1 & 11.681000000 & 5.764200000 & 6.931000000 \\ 8 & -4.854100000 & -1.138300000 & 11.280600000 \\ 1 & -5.163700000 & -0.664900000 & 10.441000000 \\ 1 & -4.584800000 & -0.465000000 & 11.984400000 \\ 8 & -8.181000000 & 2.967100000 & 0.180900000 \\ 1 & -8.589800000 & 2.961500000 & 1.108100000 \\ 1 & -8.290800000 & 2.058000000 & -0.250900000 \\ 8 & 11.723300000 & 4.627800000 & 0.096500000 \\ 1 & 10.984300000 & 4.579500000 & 0.784400000 \\ 1 & 11.364300000 & 4.378600000 & -0.814000000 \\ 8 & -6.805000000 & -6.613400000 & 6.105600000 \\ 1 & -6.332600000 & -7.167600000 & 6.808600000 \\ 1 & -7.237500000 & -5.844700000 & 6.603100000 \\ 8 & 7.742000000 & 5.415800000 & -11.744000000 \\ 1 & 7.629500000 & 5.057100000 & -12.679600000 \\ 1 & 8.114800000 & 4.664300000 & -11.180200000 \\ 8 & 8.603500000 & 3.473600000 & -9.975700000 \\ 1 & 9.403000000 & 3.019200000 & -9.552800000 \\ 1 & 7.959700000 & 3.822300000 & -9.277200000 \\ 8 & 1.934100000 & 6.965300000 & -12.512100000 \\ 1 & 2.212500000 & 6.138000000 & -13.019000000 \\ 1 & 2.767200000 & 7.377100000 & -12.117400000 \\ 8 & 1.186400000 & 5.473700000 & -8.463600000\end{array}$




$\begin{array}{cccc}1 & 0.577400000 & 4.970000000 & -9.098900000 \\ 1 & 1.572200000 & 4.811100000 & -7.802400000 \\ 8 & -4.842500000 & 0.621800000 & -14.461200000 \\ 1 & -4.211600000 & 1.371100000 & -14.220000000 \\ 1 & -5.775400000 & 0.988100000 & -14.594200000 \\ 8 & -0.427200000 & -7.439500000 & 10.614200000 \\ 1 & -0.075900000 & -6.527200000 & 10.354100000 \\ 1 & 0.340500000 & -8.092800000 & 10.689400000 \\ 8 & -10.874800000 & -6.881900000 & 4.370800000 \\ 1 & -10.874300000 & -7.869100000 & 4.590400000 \\ 1 & -11.293400000 & -6.350100000 & 5.122700000 \\ 8 & -9.406600000 & 2.608100000 & 5.695600000 \\ 1 & -8.590900000 & 3.146400000 & 5.437400000 \\ 1 & -9.265600000 & 1.624800000 & 5.499100000 \\ 8 & -7.995500000 & -3.356100000 & -2.229500000 \\ 1 & -7.812800000 & -4.163000000 & -2.811500000 \\ 1 & -8.587800000 & -2.707200000 & -2.733800000 \\ 8 & 3.003800000 & 8.441600000 & -2.951600000 \\ 1 & 3.915300000 & 8.167800000 & -2.606800000 \\ 1 & 3.097600000 & 8.760900000 & -3.908600000 \\ 8 & -4.565500000 & -4.844100000 & 0.274900000 \\ 1 & -4.916500000 & -4.992200000 & -0.662400000 \\ 1 & -4.512400000 & -5.740700000 & 0.740100000 \\ 8 & 6.513900000 & -3.303800000 & -0.484900000 \\ 1 & 7.280800000 & -3.715600000 & -1.002200000 \\ 1 & 6.547800000 & -2.304200000 & -0.641400000 \\ 8 & 7.256000000 & 4.387400000 & -7.543200000 \\ 1 & 7.854500000 & 4.242600000 & -6.739900000 \\ 1 & 7.032000000 & 5.371000000 & -7.626700000 \\ 8 & -7.817800000 & 1.836800000 & 10.054700000 \\ 1 & -8.391500000 & 1.523300000 & 10.825400000 \\ 1 & -8.463100000 & 2.260300000 & 9.398000000 \\ 8 & 10.344100000 & 0.342300000 & 5.894600000 \\ 1 & 10.384000000 & -0.376300000 & 5.183500000 \\ 1 & 11.284000000 & 0.562300000 & 6.199500000 \\ 8 & -9.432500000 & 11.978800000 & 4.676800000 \\ 1 & -10.210300000 & 12.297500000 & 4.110600000 \\ 1 & -8.887700000 & 12.757500000 & 4.996800000 \\ 8 & 7.476700000 & -7.497300000 & 0.619500000 \\ 1 & 7.998000000 & -6.945000000 & 1.290200000 \\ 1 & 7.334700000 & -8.415900000 & 1.021400000 \\ 8 & 5.035700000 & 5.673300000 & 0.593700000 \\ 1 & 4.340100000 & 6.357600000 & 0.852500000 \\ 1 & 5.854400000 & 6.207100000 & 0.332900000 \\ 1 & -9.350100000 & -3.874900000 & 0.038100000 \\ & -8.689400000 & -3.712200000 & -0.710600000\end{array}$




$\begin{array}{lccc}1 & -10.172000000 & -4.315000000 & -0.356600000 \\ 8 & 6.581100000 & 6.951200000 & -8.115600000 \\ 1 & 6.655500000 & 7.674300000 & -7.411500000 \\ 1 & 6.781500000 & 7.341600000 & -9.027400000 \\ 8 & 0.128300000 & -9.265700000 & 3.000500000 \\ 1 & 0.175500000 & -8.801200000 & 2.102000000 \\ 1 & 1.035300000 & -9.671200000 & 3.191100000 \\ 8 & -0.489700000 & 6.346500000 & -13.909300000 \\ 1 & -0.925100000 & 7.219000000 & -14.143000000 \\ 1 & 0.432200000 & 6.483700000 & -13.505800000 \\ 8 & 3.352900000 & -2.236200000 & -2.731400000 \\ 1 & 3.746300000 & -3.121200000 & -2.426000000 \\ 1 & 4.135800000 & -1.648600000 & -2.987300000 \\ 8 & -5.325900000 & -4.981800000 & -11.978600000 \\ 1 & -4.417900000 & -4.815300000 & -11.556400000 \\ 1 & -5.513100000 & -5.971900000 & -11.892600000 \\ 8 & 0.815000000 & -8.352700000 & 6.727700000 \\ 1 & 1.649800000 & -8.476000000 & 7.285700000 \\ 1 & 0.464000000 & -9.280700000 & 6.525400000 \\ 8 & 0.048500000 & -8.940700000 & -1.752400000 \\ 1 & -0.728800000 & -9.588400000 & -1.697600000 \\ 1 & 0.789400000 & -9.385000000 & -2.279800000 \\ 8 & -2.783400000 & -1.479300000 & 8.015000000 \\ 1 & -2.816400000 & -2.123000000 & 8.793800000 \\ 1 & -3.109500000 & -0.572400000 & 8.322300000 \\ 8 & 7.414400000 & -9.684700000 & 4.717600000 \\ 1 & 6.831300000 & -8.909700000 & 5.010200000 \\ 1 & 7.510700000 & -10.293600000 & 5.518900000 \\ 8 & 2.614300000 & 1.363500000 & -3.914900000 \\ 1 & 3.447900000 & 1.919500000 & -4.032900000 \\ 1 & 2.223900000 & 1.191400000 & -4.831100000 \\ 8 & -11.239200000 & 12.226300000 & 2.500900000 \\ 1 & -12.002500000 & 12.848400000 & 2.311100000 \\ 1 & -10.732800000 & 12.078900000 & 1.631500000 \\ 8 & 1.193200000 & -2.723800000 & -4.054200000 \\ 1 & 1.354800000 & -3.254300000 & -4.902300000 \\ 1 & 2.079500000 & -2.583500000 & -3.585900000 \\ 8 & 8.795000000 & -5.997900000 & 2.369500000 \\ 1 & 9.611300000 & -5.781100000 & 1.812000000 \\ 1 & 8.625600000 & -5.213000000 & 2.987300000 \\ 8 & -4.269900000 & 7.407700000 & -0.068900000 \\ 1 & -4.135000000 & 8.195700000 & 0.546500000 \\ 1 & -5.212900000 & 7.067500000 & 0.064400000 \\ 1 & -7.323800000 & -1.208100000 & 7.009100000 \\ 1 & -8.185100000 & -1.688600000 & 7.237800000\end{array}$




\begin{tabular}{cccc}
8 & -14.433600000 & 7.138200000 & 0.806000000 \\
1 & -14.099300000 & 7.510400000 & 1.686100000 \\
1 & -15.374900000 & 7.459100000 & 0.676700000 \\
8 & -3.832800000 & -14.107200000 & -0.276500000 \\
1 & -3.979300000 & -14.206600000 & 0.719300000 \\
1 & -2.902300000 & -13.758600000 & -0.472500000 \\
8 & -0.198500000 & -2.171400000 & 6.710900000 \\
1 & -0.340900000 & -3.173200000 & 6.725200000 \\
1 & -1.014800000 & -1.688000000 & 7.059500000 \\
8 & 5.712000000 & -4.339900000 & 7.814800000 \\
1 & 5.909700000 & -3.355500000 & 7.930200000 \\
1 & 5.033800000 & -4.457300000 & 7.074300000 \\
8 & 2.077800000 & -0.964900000 & 5.694700000 \\
1 & 1.410000000 & -1.502700000 & 6.224200000 \\
1 & 2.061300000 & -0.014100000 & 6.035300000 \\
8 & -4.140900000 & 9.403400000 & -7.746700000 \\
1 & -3.202900000 & 9.787600000 & -7.712300000 \\
1 & -4.309200000 & 8.874400000 & -6.900100000 \\
8 & 5.758600000 & -7.641600000 & 5.549100000 \\
1 & 5.247100000 & -7.164900000 & 4.820900000 \\
1 & 5.374800000 & -7.330000000 & 6.432800000 \\
8 & 8.189100000 & 1.782600000 & -3.875800000 \\
1 & 8.404000000 & 0.828000000 & -3.627800000 \\
1 & 7.472300000 & 2.137400000 & -3.259700000 \\
8 & 13.690500000 & 0.967600000 & -4.810900000 \\
1 & 14.119800000 & 1.564100000 & -5.505000000 \\
1 & 13.345800000 & 0.130000000 & -5.260400000 \\
8 & -9.996000000 & 7.096500000 & 2.505400000 \\
1 & -10.147400000 & 8.043300000 & 2.822100000 \\
1 & -9.078300000 & 6.759800000 & 2.766300000 \\
8 & -11.718800000 & -6.846100000 & 1.813900000 \\
1 & -11.430800000 & -6.758500000 & 2.778400000 \\
1 & -11.019800000 & -7.377700000 & 1.315100000 \\
8 & 11.853000000 & 1.221100000 & -11.926800000 \\
1 & 12.346200000 & 1.089400000 & -11.064900000 \\
1 & 12.419100000 & 0.891900000 & -12.696800000 \\
8 & -0.507100000 & 4.106800000 & -10.109500000 \\
1 & -1.125600000 & 4.525300000 & -10.792800000 \\
1 & -1.001500000 & 3.334600000 & -9.684000000 \\
8 & -9.566900000 & -2.461600000 & 7.736800000 \\
1 & -10.288900000 & -2.499200000 & 7.029000000 \\
1 & -9.980800000 & -2.419200000 & 8.657900000 \\
8 & 4.954300000 & 1.136700000 & -13.231900000 \\
& 5.904500000 & 1.083000000 & -13.572600000 \\
\hline & -0.765600000 & 2.113400000 & -13.044400000 \\
\hline & & & \\
1 & & &
\end{tabular}




$\begin{array}{cccc}1 & -0.396700000 & -11.160400000 & 6.994000000 \\ 1 & -0.778000000 & -10.966800000 & 5.393000000 \\ 8 & -4.049600000 & -3.346000000 & 4.524800000 \\ 1 & -4.574200000 & -3.416500000 & 5.383900000 \\ 1 & -4.694900000 & -3.303100000 & 3.749000000 \\ 8 & -3.698400000 & -9.832000000 & 8.088200000 \\ 1 & -3.679400000 & -10.249300000 & 7.164600000 \\ 1 & -2.845100000 & -9.311400000 & 8.242600000 \\ 8 & 0.884900000 & -5.073000000 & 9.885600000 \\ 1 & 1.853300000 & -5.208400000 & 10.147400000 \\ 1 & 0.623500000 & -4.169800000 & 10.250200000 \\ 8 & 1.069500000 & 11.654000000 & -2.187700000 \\ 1 & 1.862800000 & 12.144500000 & -1.803900000 \\ 1 & 1.069300000 & 11.823100000 & -3.184200000 \\ 8 & 10.595300000 & -2.852600000 & -8.759600000 \\ 1 & 10.108700000 & -1.985100000 & -8.569900000 \\ 1 & 9.903700000 & -3.541800000 & -9.004700000 \\ 8 & 8.271300000 & -4.940100000 & 6.668800000 \\ 1 & 7.408200000 & -4.784500000 & 7.171600000 \\ 1 & 8.527000000 & -5.917300000 & 6.719800000 \\ 8 & -6.313700000 & -1.589500000 & -1.001800000 \\ 1 & -5.433800000 & -1.655100000 & -1.498900000 \\ 1 & -6.901200000 & -2.334500000 & -1.353800000 \\ 8 & -3.797500000 & 0.881100000 & 0.744900000 \\ 1 & -4.502900000 & 0.219300000 & 1.035900000 \\ 1 & -4.226700000 & 1.774600000 & 0.554800000 \\ 8 & 2.453200000 & 0.508600000 & -14.437400000 \\ 1 & 3.409700000 & 0.742000000 & -14.203800000 \\ 1 & 2.049500000 & 1.258800000 & -14.982000000 \\ 8 & -11.061500000 & -2.257500000 & 10.195400000 \\ 1 & -11.614400000 & -1.411500000 & 10.183800000 \\ 1 & -10.399300000 & -2.173600000 & 10.955600000 \\ 8 & 10.994400000 & -4.716100000 & 1.310900000 \\ 1 & 11.739700000 & -4.922500000 & 1.965200000 \\ 1 & 11.073200000 & -3.756700000 & 1.007100000 \\ 8 & 0.835000000 & 7.549600000 & -10.118000000 \\ 1 & 1.274400000 & 7.450800000 & -11.022700000 \\ 1 & 1.170000000 & 6.793400000 & -9.535100000 \\ 8 & -10.848600000 & -3.669400000 & -7.648800000 \\ 1 & -9.888400000 & -3.569300000 & -7.957000000 \\ 1 & -11.455600000 & -3.883500000 & -8.417900000 \\ 8 & 7.660900000 & 12.982000000 & -0.139500000 \\ 1 & 7.201500000 & 12.214400000 & -0.613900000 \\ 8 & 8.666300000 & 12.922700000 & -0.259200000 \\ 1 & -7.758400000 & 0.805900000 & -14.624300000 \\ & -8.268500000 & 0.537900000 & -15.445200000\end{array}$




$\begin{array}{cccc}1 & -7.582700000 & -0.041200000 & -14.090200000 \\ 8 & 3.325100000 & -2.525000000 & -11.325500000 \\ 1 & 3.528800000 & -3.488500000 & -11.092600000 \\ 1 & 2.950700000 & -2.466800000 & -12.262500000 \\ 8 & 8.828000000 & 10.003500000 & -5.113000000 \\ 1 & 9.678400000 & 10.052900000 & -5.655900000 \\ 1 & 8.994900000 & 10.234600000 & -4.152800000 \\ 8 & -6.154600000 & 11.425900000 & 0.339500000 \\ 1 & -6.733900000 & 10.681100000 & 0.699000000 \\ 1 & -6.736500000 & 12.199800000 & 0.041100000 \\ 8 & -8.705900000 & -0.456300000 & -7.929900000 \\ 1 & -9.507900000 & 0.093400000 & -7.651200000 \\ 1 & -8.091800000 & 0.160000000 & -8.448900000 \\ 8 & -4.749800000 & -0.767400000 & -5.548500000 \\ 1 & -4.292100000 & -0.526000000 & -4.677200000 \\ 1 & -5.733700000 & -0.541600000 & -5.459200000 \\ 8 & -4.101300000 & 9.659200000 & 1.581600000 \\ 1 & -4.381600000 & 9.639200000 & 2.554700000 \\ 1 & -4.653700000 & 10.330100000 & 1.066300000 \\ 8 & -6.935200000 & -2.986800000 & 11.442000000 \\ 1 & -6.013300000 & -2.593500000 & 11.405100000 \\ 1 & -6.823700000 & -3.996800000 & 11.462400000 \\ 8 & -8.581500000 & -8.996000000 & 9.982100000 \\ 1 & -7.604100000 & -8.829600000 & 10.188200000 \\ 1 & -8.915400000 & -9.700900000 & 10.612800000 \\ 8 & 6.573800000 & 12.179700000 & 6.327700000 \\ 1 & 7.155900000 & 12.786900000 & 5.764300000 \\ 1 & 6.525900000 & 12.535400000 & 7.264500000 \\ 8 & -10.681800000 & 0.751300000 & -6.473900000 \\ 1 & -10.603300000 & 1.344100000 & -5.657500000 \\ 1 & -10.874400000 & 1.351700000 & -7.264200000 \\ 8 & -14.889100000 & -1.084900000 & 2.440600000 \\ 1 & -15.612800000 & -1.549600000 & 2.969500000 \\ 1 & -14.490900000 & -0.323900000 & 2.969700000 \\ 8 & 6.950600000 & -0.125100000 & 2.066600000 \\ 1 & 7.150500000 & -0.338800000 & 1.097400000 \\ 1 & 6.973800000 & -0.991200000 & 2.588800000 \\ 8 & -0.728300000 & -3.654100000 & -11.805500000 \\ 1 & -0.920200000 & -3.509900000 & -12.786400000 \\ 1 & -0.402200000 & -2.785300000 & -11.406600000 \\ 8 & -5.289000000 & 4.686900000 & -10.115300000 \\ 1 & -5.346200000 & 3.998700000 & -10.855400000 \\ 1 & -4.513000000 & 5.310400000 & -10.297400000 \\ 8 & 8.813200000 & 3.993300000 & -5.282200000 \\ 1 & 8.699800000 & 4.759700000 & -4.633500000 \\ 1 & 8.578100000 & 3.155000000 & -4.761400000\end{array}$




$\begin{array}{cccc}8 & 3.071100000 & -8.526700000 & -0.322800000 \\ 1 & 3.695700000 & -8.782700000 & -1.075800000 \\ 1 & 2.661500000 & -7.619100000 & -0.502700000 \\ 8 & 2.565500000 & -0.263300000 & 0.932500000 \\ 1 & 3.465200000 & 0.160100000 & 0.698300000 \\ 1 & 2.664700000 & -0.750900000 & 1.818800000 \\ 8 & -11.415300000 & 0.913900000 & 1.548400000 \\ 1 & -11.224500000 & 0.001200000 & 1.156400000 \\ 1 & -12.057300000 & 1.416500000 & 0.950000000 \\ 8 & -11.032000000 & 5.450600000 & -1.656800000 \\ 1 & -11.378900000 & 6.057400000 & -0.924200000 \\ 1 & -11.285500000 & 5.880100000 & -2.536100000 \\ 8 & 1.277700000 & -5.733400000 & 5.603900000 \\ 1 & 0.673300000 & -5.174800000 & 6.199500000 \\ 1 & 1.093100000 & -6.715300000 & 5.765600000 \\ 8 & -3.882000000 & -10.655000000 & -7.599800000 \\ 1 & -4.599300000 & -10.731600000 & -6.889200000 \\ 1 & -3.676600000 & -11.573300000 & -7.970100000 \\ 8 & 7.463300000 & -0.701900000 & -10.150000000 \\ 1 & 8.014900000 & -0.068800000 & -10.712500000 \\ 1 & 6.498300000 & -0.582700000 & -10.428400000 \\ 8 & 3.067600000 & 7.659800000 & 1.218800000 \\ 1 & 2.529100000 & 8.027200000 & 0.441500000 \\ 1 & 3.121900000 & 8.371400000 & 1.937900000 \\ 8 & 2.905900000 & 13.504100000 & -7.373400000 \\ 1 & 2.381600000 & 14.304800000 & -7.674700000 \\ 1 & 3.147800000 & 12.961900000 & -8.193500000 \\ 8 & -5.981800000 & -10.456100000 & -5.869100000 \\ 1 & -5.874000000 & -10.849600000 & -4.945700000 \\ 1 & -6.849200000 & -9.936700000 & -5.918300000 \\ 8 & -0.070400000 & 1.556400000 & -2.780600000 \\ 1 & -0.897200000 & 1.907200000 & -3.250900000 \\ 1 & 0.692600000 & 1.495100000 & -3.447000000 \\ 8 & -3.384800000 & 3.554300000 & 9.428500000 \\ 1 & -2.867600000 & 4.358900000 & 9.755700000 \\ 1 & -4.373700000 & 3.706600000 & 9.564200000 \\ 8 & 5.442600000 & 7.711800000 & 3.915300000 \\ 1 & 4.455200000 & 7.545900000 & 3.770900000 \\ 1 & 5.630900000 & 7.659800000 & 4.907800000 \\ 8 & 2.183200000 & -2.610300000 & 12.231300000 \\ 1 & 2.283800000 & -1.600800000 & 12.220500000 \\ 1 & 1.267000000 & -2.858300000 & 11.885400000 \\ & -6.355000000 & -13.234400000 & 7.896900000 \\ 1 & 3.182300000 & -1.795100000 & 8.980700000\end{array}$




$\begin{array}{cccc}1 & 2.281000000 & -1.552300000 & 9.379600000 \\ 1 & 3.595000000 & -2.491700000 & 9.590800000 \\ 8 & 5.772200000 & 15.172300000 & -3.913300000 \\ 1 & 5.730900000 & 16.173100000 & -3.903900000 \\ 1 & 5.740600000 & 14.840000000 & -2.959200000 \\ 8 & 0.483000000 & -7.105300000 & -9.371900000 \\ 1 & -0.497100000 & -7.330400000 & -9.481700000 \\ 1 & 0.837000000 & -6.896700000 & -10.297000000 \\ 8 & -1.989800000 & -10.704700000 & -1.600800000 \\ 1 & -2.391800000 & -10.917300000 & -2.504700000 \\ 1 & -1.698600000 & -11.576700000 & -1.178500000 \\ 8 & 3.771300000 & -12.063900000 & -4.060200000 \\ 1 & 4.446300000 & -11.788800000 & -3.353600000 \\ 1 & 3.873900000 & -11.456100000 & -4.859700000 \\ 8 & 2.688300000 & 7.045300000 & 4.561600000 \\ 1 & 1.854900000 & 7.298900000 & 4.055200000 \\ 1 & 2.604000000 & 6.068700000 & 4.816100000 \\ 8 & 4.693800000 & 0.295900000 & -8.191500000 \\ 1 & 4.937600000 & 0.002100000 & -9.129300000 \\ 1 & 3.836900000 & -0.191000000 & -7.963200000 \\ 8 & -8.250400000 & -3.065000000 & -8.439600000 \\ 1 & -8.420500000 & -2.099700000 & -8.184500000 \\ 1 & -7.731700000 & -3.116100000 & -9.307400000 \\ 8 & 2.172600000 & -8.199600000 & -7.356700000 \\ 1 & 1.618800000 & -7.941100000 & -8.163900000 \\ 1 & 1.555700000 & -8.542500000 & -6.631300000 \\ 8 & 2.943600000 & 11.648600000 & -9.339200000 \\ 1 & 2.924700000 & 11.780100000 & -10.333500000 \\ 1 & 2.201300000 & 11.005300000 & -9.085900000 \\ 8 & 9.139700000 & 1.266000000 & -11.606600000 \\ 1 & 8.916900000 & 2.124800000 & -11.121100000 \\ 1 & 10.144600000 & 1.178100000 & -11.715700000 \\ 8 & 6.761600000 & 14.324400000 & 2.150200000 \\ 1 & 6.569200000 & 15.279900000 & 1.881100000 \\ 1 & 7.186700000 & 13.818900000 & 1.381400000 \\ 8 & 7.595100000 & 1.295500000 & -13.847500000 \\ 1 & 8.158100000 & 1.287200000 & -13.004700000 \\ 1 & 8.018400000 & 0.638300000 & -14.475900000 \\ 8 & 5.257200000 & 9.620800000 & 0.227300000 \\ 1 & 5.893900000 & 9.325900000 & 0.957000000 \\ 1 & 5.106200000 & 8.847400000 & -0.408600000 \\ 8 & -4.306200000 & 5.201200000 & -5.815800000 \\ 1 & -4.073800000 & 4.980200000 & -4.857400000 \\ 1 & -5.181600000 & 4.765900000 & -6.078900000 \\ & -1.424800000 & 8.065000000 & -3.893500000 \\ & -1.240800000 & 8.985600000 & -4.271200000\end{array}$




\begin{tabular}{lrrr}
1 & -2.346300000 & 8.043100000 & -3.474400000 \\
8 & 1.439800000 & -10.730300000 & -3.516100000 \\
1 & 0.738600000 & -11.449800000 & -3.635000000 \\
1 & 2.328700000 & -11.182900000 & -3.691500000 \\
8 & -3.144000000 & -0.864200000 & 5.379000000 \\
1 & -2.957500000 & -1.047000000 & 6.355600000 \\
1 & -3.506600000 & -1.707700000 & 4.948200000 \\
8 & 6.348600000 & -12.113100000 & 0.729700000 \\
1 & 7.090100000 & -12.787000000 & 0.696400000 \\
1 & 5.508800000 & -12.527300000 & 1.125800000 \\
8 & 1.307300000 & 4.444200000 & -1.933700000 \\
1 & 1.523400000 & 4.562700000 & -2.915900000 \\
1 & 0.772500000 & 5.257400000 & -1.654000000 \\
8 & -0.535300000 & -7.948900000 & 0.674600000 \\
1 & -1.494700000 & -8.269500000 & 0.621800000 \\
1 & -0.076300000 & -8.253300000 & -0.174000000 \\
8 & -7.883700000 & -4.879500000 & 7.674400000 \\
1 & -8.328800000 & -5.374800000 & 8.435600000 \\
1 & -8.299100000 & -3.967900000 & 7.544000000 \\
8 & 1.292600000 & -5.544300000 & -11.572500000 \\
1 & 0.617300000 & -4.792800000 & -11.531800000 \\
1 & 1.823600000 & -5.477200000 & -12.433300000 \\
8 & 0.668200000 & -1.420700000 & -10.890500000 \\
1 & 1.603600000 & -1.806400000 & -10.851900000 \\
1 & 0.744800000 & -0.481100000 & -11.261400000 \\
8 & 6.949500000 & -6.461200000 & -7.901400000 \\
1 & 7.800800000 & -6.327600000 & -7.373400000 \\
1 & 7.125700000 & -6.149300000 & -8.837600000 \\
8 & -4.120000000 & -2.770200000 & -9.578000000 \\
1 & -3.593000000 & -3.573800000 & -9.893800000 \\
1 & -3.924500000 & -1.999100000 & -10.204600000 \\
8 & 13.069500000 & -5.096100000 & 3.082200000 \\
1 & 12.789300000 & -5.901600000 & 3.625300000 \\
1 & 13.008100000 & -4.285100000 & 3.682200000 \\
8 & -1.785600000 & -4.480500000 & 3.283600000 \\
1 & -1.757800000 & -5.449100000 & 3.571100000 \\
1 & -2.596300000 & -4.036300000 & 3.696800000 \\
8 & 8.866200000 & 0.897400000 & -0.302500000 \\
1 & 9.664400000 & 0.845300000 & -0.923400000 \\
1 & 8.480800000 & 1.833400000 & -0.295000000 \\
8 & -7.707100000 & 9.992700000 & 3.773400000 \\
1 & -7.811100000 & 9.706400000 & 2.807500000 \\
1 & -8.337900000 & 10.765300000 & 3.939200000 \\
1 & -5.991200000 & 4.881800000 & 7.077100000 \\
& -5.154200000 & 4.733900000 & 8.065000000 \\
\hline & & & \\
1 & &
\end{tabular}




$\begin{array}{cccc}8 & 6.003700000 & 1.999700000 & 7.174900000 \\ 1 & 5.150700000 & 1.480500000 & 7.338100000 \\ 1 & 6.554800000 & 2.033000000 & 8.023100000 \\ 8 & -0.785300000 & 10.198200000 & -1.139400000 \\ 1 & -0.121200000 & 10.875400000 & -1.498700000 \\ 1 & -1.708900000 & 10.345700000 & -1.522100000 \\ 8 & -1.394600000 & -7.328500000 & -3.384400000 \\ 1 & -2.335200000 & -7.687000000 & -3.295900000 \\ 1 & -0.785500000 & -7.761000000 & -2.701400000 \\ 8 & 16.734900000 & -2.439100000 & -2.067400000 \\ 1 & 17.718700000 & -2.225300000 & -2.169600000 \\ 1 & 16.523200000 & -2.534900000 & -1.091700000 \\ 8 & 5.986100000 & 7.896700000 & 6.621700000 \\ 1 & 6.241000000 & 7.056600000 & 7.123800000 \\ 1 & 5.010500000 & 8.099900000 & 6.793800000 \\ 8 & 5.010500000 & -6.960500000 & 8.258100000 \\ 1 & 5.819600000 & -7.552200000 & 8.389000000 \\ 1 & 5.313400000 & -6.003200000 & 8.158400000 \\ 8 & -6.984700000 & 7.339700000 & 10.185600000 \\ 1 & -7.869100000 & 7.820700000 & 10.275900000 \\ 1 & -6.927800000 & 6.591600000 & 10.868200000 \\ 8 & -1.488100000 & -7.022200000 & 3.917200000 \\ 1 & -1.865200000 & -7.084800000 & 4.854000000 \\ 1 & -1.018700000 & -7.897500000 & 3.721200000 \\ 8 & -2.890900000 & -7.489700000 & 11.714000000 \\ 1 & -1.923000000 & -7.310700000 & 11.522900000 \\ 1 & -3.074300000 & -8.488600000 & 11.673700000 \\ 8 & -10.365100000 & 2.568000000 & -4.515200000 \\ 1 & -10.907200000 & 2.621700000 & -3.662000000 \\ 1 & -9.410300000 & 2.314000000 & -4.294300000 \\ 8 & 7.348600000 & 4.111000000 & -14.081300000 \\ 1 & 7.811400000 & 4.547000000 & -14.869500000 \\ 1 & 7.437300000 & 3.117700000 & -14.189900000 \\ 8 & -5.186900000 & 2.940200000 & 3.357700000 \\ 1 & -4.584700000 & 2.506300000 & 4.046800000 \\ 1 & -4.648300000 & 3.607500000 & 2.818600000 \\ 8 & 10.635900000 & 8.056300000 & 1.356000000 \\ 1 & 11.558000000 & 7.644000000 & 1.412600000 \\ 1 & 10.035500000 & 7.548300000 & 1.992400000 \\ 8 & -9.681300000 & -1.811300000 & 3.529200000 \\ 1 & -10.108100000 & -1.500000000 & 2.666600000 \\ 1 & -9.109200000 & -2.623700000 & 3.345400000 \\ 8 & -6.156900000 & -0.086000000 & 9.129400000 \\ 1 & -6.822700000 & 0.574600000 & 9.511700000 \\ 8 & -6.606800000 & -0.612900000 & 8.390100000 \\ & 0.487300000 & -8.977800000 & -5.359400000\end{array}$




\begin{tabular}{cccc}
1 & 0.800200000 & -9.712800000 & -4.736600000 \\
1 & -0.481000000 & -9.161500000 & -5.592600000 \\
8 & 9.486600000 & 7.594600000 & 5.723100000 \\
1 & 8.965600000 & 8.453500000 & 5.831200000 \\
1 & 8.995300000 & 6.873100000 & 6.229700000 \\
8 & -11.417500000 & -2.592200000 & 5.710700000 \\
1 & -12.131700000 & -1.888400000 & 5.843500000 \\
1 & -10.979400000 & -2.372100000 & 4.824700000 \\
8 & 3.961100000 & 15.658900000 & 2.452400000 \\
1 & 3.492700000 & 16.074600000 & 3.250400000 \\
1 & 4.168100000 & 14.695500000 & 2.639800000 \\
8 & 2.693900000 & 3.247200000 & 1.668100000 \\
1 & 2.494200000 & 2.788500000 & 2.548200000 \\
1 & 3.695200000 & 3.395600000 & 1.602700000 \\
8 & -3.249600000 & -0.992500000 & -11.447800000 \\
1 & -2.480800000 & -0.336500000 & -11.403900000 \\
1 & -3.291000000 & -1.381900000 & -12.380400000 \\
8 & -7.349300000 & -7.273600000 & -1.293000000 \\
1 & -7.194800000 & -7.185400000 & -0.296900000 \\
1 & -8.324800000 & -7.077200000 & -1.462800000 \\
8 & 6.894900000 & -10.943400000 & 7.181500000 \\
1 & 7.442400000 & -11.781900000 & 7.241100000 \\
1 & 5.923300000 & -11.225000000 & 7.243700000 \\
8 & -10.005300000 & -6.973300000 & -1.500200000 \\
1 & -10.006100000 & -7.473700000 & -0.621700000 \\
1 & -10.620000000 & -6.176900000 & -1.391800000 \\
8 & 0.860400000 & -0.434900000 & 9.972500000 \\
1 & 0.160900000 & 0.202500000 & 9.600700000 \\
1 & 1.366700000 & 0.057500000 & 10.695700000 \\
8 & 14.455900000 & -2.640200000 & 8.908800000 \\
1 & 13.958700000 & -3.136400000 & 8.182500000 \\
1 & 15.445200000 & -2.720100000 & 8.771000000 \\
8 & -6.068600000 & 3.914900000 & 9.746000000 \\
1 & -6.597000000 & 3.057300000 & 9.829300000 \\
1 & -6.367300000 & 4.556200000 & 10.468500000 \\
8 & 4.000300000 & -9.900800000 & -5.930000000 \\
1 & 4.967700000 & -9.653400000 & -6.087700000 \\
1 & 3.393500000 & -9.361000000 & -6.529000000 \\
8 & 5.727200000 & 14.609200000 & -1.012600000 \\
1 & 6.513400000 & 13.995400000 & -0.838800000 \\
1 & 5.792700000 & 15.400300000 & -0.390000000 \\
8 & -3.455100000 & -3.861200000 & -4.124600000 \\
1 & -3.709400000 & -3.753700000 & -5.097400000 \\
1 & -2.524000000 & -4.267200000 & -4.099500000 \\
1 & -2.134800000 & 2.546600000 & -4.136100000 \\
\hline & -2.872400000 & 3.217400000 & -3.966200000
\end{tabular}




$\begin{array}{cccc}1 & -1.699800000 & 2.760100000 & -5.022900000 \\ 8 & 11.515200000 & 2.551200000 & -3.985700000 \\ 1 & 11.161200000 & 2.640400000 & -4.926500000 \\ 1 & 12.380600000 & 2.037800000 & -4.048600000 \\ 8 & 7.270000000 & 7.311100000 & 0.051600000 \\ 1 & 7.374100000 & 7.972300000 & 0.807800000 \\ 1 & 8.125200000 & 7.287200000 & -0.485200000 \\ 8 & 0.061300000 & -2.152600000 & -8.356500000 \\ 1 & -0.340100000 & -3.081500000 & -8.395200000 \\ 1 & 0.203300000 & -1.843300000 & -9.310400000 \\ 8 & 2.824300000 & 4.466400000 & 5.263600000 \\ 1 & 2.987900000 & 4.541400000 & 6.260200000 \\ 1 & 3.712300000 & 4.193300000 & 4.863500000 \\ 8 & -9.710500000 & 0.871500000 & 11.872800000 \\ 1 & -9.434000000 & -0.051200000 & 12.175300000 \\ 1 & -10.684900000 & 0.855700000 & 11.601900000 \\ 8 & -8.155500000 & 6.206200000 & -6.367000000 \\ 1 & -9.131800000 & 5.982400000 & -6.508000000 \\ 1 & -7.723500000 & 6.459400000 & -7.246200000 \\ 8 & 4.390400000 & -8.202900000 & 2.072900000 \\ 1 & 5.267500000 & -8.655400000 & 1.847900000 \\ 1 & 3.878600000 & -8.088500000 & 1.207300000 \\ 8 & 13.310300000 & 2.619700000 & 1.252900000 \\ 1 & 13.514800000 & 1.913300000 & 0.557400000 \\ 1 & 12.664500000 & 3.299900000 & 0.875800000 \\ 8 & 8.238100000 & 3.601600000 & -0.556400000 \\ 1 & 8.548200000 & 4.053500000 & 0.298400000 \\ 1 & 8.947200000 & 3.789800000 & -1.247700000 \\ 8 & 8.375200000 & 2.323200000 & 5.508300000 \\ 1 & 7.550700000 & 1.990400000 & 5.990800000 \\ 1 & 9.134300000 & 1.692600000 & 5.740000000 \\ 8 & 6.512800000 & -5.300000000 & -10.604000000 \\ 1 & 6.716200000 & -5.803200000 & -11.445700000 \\ 1 & 5.513900000 & -5.182500000 & -10.523000000 \\ 8 & -1.099700000 & 1.226600000 & 8.867900000 \\ 1 & -0.771600000 & 1.988600000 & 8.292000000 \\ 1 & -2.042200000 & 0.989000000 & 8.590200000 \\ 8 & -6.499100000 & -11.553600000 & -0.073300000 \\ 1 & -5.801800000 & -11.491100000 & 0.658900000 \\ 1 & -6.318000000 & -12.357300000 & -0.659600000 \\ 8 & -5.748600000 & -3.342600000 & 6.708600000 \\ 1 & -6.259600000 & -4.085000000 & 7.167200000 \\ 1 & -6.245600000 & -2.473900000 & 6.852800000 \\ 8 & -4.906000000 & -7.440500000 & 4.383300000 \\ 1 & -5.681600000 & -7.032200000 & 4.887800000 \\ 1 & -4.042300000 & -7.255400000 & 4.875900000\end{array}$




$\begin{array}{cccc}8 & -9.170700000 & -6.202200000 & 9.901200000 \\ 1 & -10.078400000 & -5.791000000 & 9.733800000 \\ 1 & -9.265100000 & -7.196500000 & 10.058100000 \\ 8 & 14.015800000 & -7.853200000 & 0.367300000 \\ 1 & 14.638700000 & -8.538700000 & -0.016000000 \\ 1 & 13.740600000 & -8.164100000 & 1.294100000 \\ 8 & 5.558000000 & -1.133300000 & -3.657000000 \\ 1 & 5.607500000 & -0.405000000 & -4.356900000 \\ 1 & 5.699400000 & -2.029100000 & -4.108400000 \\ 8 & 4.882200000 & -0.391400000 & -10.935200000 \\ 1 & 4.698900000 & 0.171800000 & -11.754900000 \\ 1 & 4.363500000 & -1.253900000 & -11.047900000 \\ 8 & 4.155800000 & -6.123600000 & -7.761900000 \\ 1 & 5.156300000 & -6.263900000 & -7.709200000 \\ 1 & 3.697400000 & -6.998000000 & -7.537100000 \\ 8 & -6.707900000 & 7.756400000 & 7.500300000 \\ 1 & -6.441200000 & 6.818500000 & 7.228600000 \\ 1 & -6.939000000 & 7.746600000 & 8.486100000 \\ 8 & -8.971200000 & 3.048900000 & -10.381000000 \\ 1 & -8.337100000 & 2.415400000 & -9.911500000 \\ 1 & -8.809200000 & 2.937800000 & -11.372500000 \\ 8 & -3.738400000 & 10.212000000 & 9.417600000 \\ 1 & -3.609700000 & 11.196200000 & 9.611300000 \\ 1 & -3.970500000 & 10.044200000 & 8.444400000 \\ 8 & 10.300700000 & -3.237600000 & 8.024600000 \\ 1 & 9.583600000 & -3.855100000 & 7.664900000 \\ 1 & 9.908200000 & -2.305200000 & 7.989600000 \\ 8 & -9.925800000 & 11.832700000 & 0.205000000 \\ 1 & -9.907900000 & 10.916100000 & -0.220900000 \\ 1 & -9.253800000 & 12.428700000 & -0.254800000 \\ 8 & 2.798900000 & -10.200800000 & 3.126800000 \\ 1 & 3.362000000 & -9.374500000 & 2.966200000 \\ 1 & 2.628500000 & -10.684900000 & 2.253800000 \\ 8 & -13.339300000 & 0.805000000 & 3.623300000 \\ 1 & -12.547500000 & 0.870700000 & 2.995600000 \\ 1 & -13.696000000 & 1.748300000 & 3.716900000 \\ 8 & -8.664200000 & -9.165300000 & 7.154000000 \\ 1 & -8.656200000 & -9.131300000 & 8.162900000 \\ 1 & -8.230800000 & -8.313300000 & 6.817800000 \\ 8 & -0.237300000 & -2.811500000 & 10.613700000 \\ 1 & 0.174100000 & -1.940100000 & 10.304400000 \\ 1 & -1.223000000 & -2.768200000 & 10.443500000 \\ 8 & 2.293600000 & -1.572600000 & 3.221100000 \\ 1 & 1.975100000 & -2.535300000 & 3.203500000 \\ 8 & 2.248100000 & -1.284600000 & 4.188300000 \\ & -6.686200000 & -3.082000000 & -10.675600000\end{array}$




$\begin{array}{cccc}1 & -5.852400000 & -2.777200000 & -10.192300000 \\ 1 & -6.407100000 & -3.891000000 & -11.214100000 \\ 8 & -1.385600000 & 9.105300000 & 1.382200000 \\ 1 & -1.047600000 & 9.440900000 & 0.489700000 \\ 1 & -2.349600000 & 9.391100000 & 1.506300000 \\ 8 & 14.762200000 & -4.360300000 & -1.984300000 \\ 1 & 14.827000000 & -4.707400000 & -1.036700000 \\ 1 & 15.589000000 & -3.794500000 & -2.116600000 \\ 8 & -1.363300000 & 2.595100000 & 3.161200000 \\ 1 & -1.412500000 & 3.103500000 & 2.290500000 \\ 1 & -0.765400000 & 3.138600000 & 3.770100000 \\ 8 & 6.346400000 & 2.898700000 & -2.260300000 \\ 1 & 5.461700000 & 3.286400000 & -1.954900000 \\ 1 & 7.068500000 & 3.194900000 & -1.616500000 \\ 8 & -5.087500000 & 9.487100000 & 4.187200000 \\ 1 & -4.647900000 & 9.511200000 & 5.098100000 \\ 1 & -6.021000000 & 9.878300000 & 4.222500000 \\ 8 & -0.573700000 & -4.712100000 & 7.416300000 \\ 1 & -1.575300000 & -4.776000000 & 7.530500000 \\ 1 & -0.121600000 & -4.820800000 & 8.314200000 \\ 8 & -1.897000000 & -15.265100000 & 7.277900000 \\ 1 & -2.305300000 & -16.080800000 & 7.711300000 \\ 1 & -2.594000000 & -14.535800000 & 7.224300000 \\ 8 & -7.306700000 & -5.382100000 & -4.103100000 \\ 1 & -7.993500000 & -6.123800000 & -4.074800000 \\ 1 & -7.117400000 & -5.158500000 & -5.072000000 \\ 8 & 1.744600000 & 3.419200000 & -11.247700000 \\ 1 & 0.857300000 & 3.632800000 & -10.810800000 \\ 1 & 1.790200000 & 3.892000000 & -12.141800000 \\ 8 & 11.145800000 & 0.694100000 & -1.839800000 \\ 1 & 12.039100000 & 0.589300000 & -1.374100000 \\ 1 & 11.203700000 & 1.450600000 & -2.505300000 \\ 8 & -0.371500000 & -4.775700000 & -8.166900000 \\ 1 & -1.179000000 & -5.009300000 & -7.600600000 \\ 1 & -0.037400000 & -5.620600000 & -8.614200000 \\ 8 & 6.251700000 & -8.515300000 & -1.474700000 \\ 1 & 6.571400000 & -8.241500000 & -2.394100000 \\ 1 & 6.843100000 & -8.061300000 & -0.789900000 \\ 8 & -1.321800000 & -11.641000000 & 8.520400000 \\ 1 & -2.269300000 & -11.986700000 & 8.434700000 \\ 1 & -1.155300000 & -11.268700000 & 9.445000000 \\ 8 & -7.007700000 & 6.454700000 & -8.844000000 \\ 1 & -6.429000000 & 5.710200000 & -9.208300000 \\ 8 & -6.654900000 & 7.336600000 & -9.190000000 \\ 1 & -11.711300000 & -4.900400000 & 9.277100000 \\ & -12.678800000 & -5.070700000 & 9.480300000\end{array}$




$\begin{array}{cccc}1 & -11.457000000 & -3.972400000 & 9.593400000 \\ 8 & 2.402400000 & -14.092600000 & 5.399600000 \\ 1 & 2.457600000 & -13.092400000 & 5.254900000 \\ 1 & 2.722500000 & -14.301800000 & 6.334700000 \\ 8 & 13.430300000 & 0.163700000 & -0.539800000 \\ 1 & 13.931000000 & -0.184700000 & -1.348300000 \\ 1 & 13.651900000 & -0.447600000 & 0.236300000 \\ 8 & -11.511600000 & 6.917900000 & -4.008600000 \\ 1 & -12.438500000 & 7.163200000 & -4.303300000 \\ 1 & -11.099800000 & 6.244200000 & -4.643200000 \\ 8 & 10.766300000 & -2.286000000 & 10.651500000 \\ 1 & 10.848700000 & -2.769000000 & 9.765200000 \\ 1 & 11.624200000 & -1.770400000 & 10.811700000 \\ 8 & 9.139200000 & 6.511800000 & 3.115100000 \\ 1 & 9.483000000 & 6.998700000 & 3.932600000 \\ 1 & 8.301300000 & 6.022500000 & 3.403600000 \\ 8 & -13.634600000 & 7.554100000 & 5.977600000 \\ 1 & -13.674700000 & 8.245500000 & 6.714400000 \\ 1 & -12.676800000 & 7.229400000 & 5.924200000 \\ 8 & -1.074900000 & 3.644700000 & 0.504700000 \\ 1 & -0.286000000 & 4.225700000 & 0.747700000 \\ 1 & -1.777600000 & 4.231600000 & 0.077300000 \\ 8 & 4.224400000 & -11.708800000 & 7.490900000 \\ 1 & 3.869200000 & -12.500400000 & 8.015600000 \\ 1 & 3.658500000 & -11.590900000 & 6.659900000 \\ 8 & -1.500000000 & 1.078600000 & -11.556100000 \\ 1 & -1.948900000 & 1.637500000 & -12.269800000 \\ 1 & -0.519400000 & 1.023900000 & -11.797900000 \\ 8 & 4.004800000 & 6.179100000 & -7.578900000 \\ 1 & 4.906400000 & 6.430700000 & -7.964700000 \\ 1 & 3.523100000 & 5.576700000 & -8.235700000 \\ 8 & -5.080500000 & 2.859800000 & -7.935100000 \\ 1 & -5.632900000 & 3.293400000 & -7.206200000 \\ 1 & -4.967600000 & 3.543300000 & -8.672600000 \\ 8 & -8.423300000 & 2.895600000 & -13.212300000 \\ 1 & -8.101500000 & 2.142100000 & -13.807300000 \\ 1 & -8.985600000 & 3.536200000 & -13.740700000 \\ 8 & 4.633700000 & 1.113400000 & 0.089000000 \\ 1 & 5.042700000 & 1.840800000 & 0.657800000 \\ 1 & 5.336000000 & 0.635100000 & -0.460500000 \\ 8 & 10.216200000 & -0.245300000 & 1.685500000 \\ 1 & 9.699300000 & 0.340700000 & 1.041200000 \\ 1 & 10.735800000 & -0.922300000 & 1.145500000 \\ 8 & -1.083200000 & 10.667100000 & -4.825000000 \\ 1 & -0.204000000 & 11.163500000 & -4.885700000 \\ 1 & -1.612400000 & 11.023400000 & -4.039700000\end{array}$




$\begin{array}{cccc}8 & 3.497200000 & 13.005600000 & -1.521000000 \\ 1 & 4.006500000 & 12.529400000 & -2.256000000 \\ 1 & 4.018900000 & 13.831400000 & -1.261600000 \\ 8 & 0.186900000 & 6.802500000 & -5.533600000 \\ 1 & -0.325300000 & 7.345000000 & -4.850500000 \\ 1 & -0.473200000 & 6.297800000 & -6.110200000 \\ 8 & 1.709200000 & 4.830500000 & -4.575500000 \\ 1 & 1.662700000 & 4.124400000 & -5.297000000 \\ 1 & 1.073600000 & 5.579400000 & -4.822400000 \\ 8 & -13.477800000 & -2.797200000 & 0.766800000 \\ 1 & -13.749400000 & -3.772000000 & 0.731900000 \\ 1 & -13.943000000 & -2.310900000 & 1.520900000 \\ 8 & 14.715400000 & 3.352400000 & 3.577700000 \\ 1 & 14.142300000 & 4.155000000 & 3.786600000 \\ 1 & 14.542400000 & 3.102000000 & 2.614900000 \\ 8 & 10.537100000 & 12.170500000 & -0.719500000 \\ 1 & 11.333700000 & 12.751200000 & -0.898000000 \\ 1 & 10.655300000 & 11.746400000 & 0.189900000 \\ 8 & 0.637600000 & 8.397100000 & 3.509500000 \\ 1 & -0.132100000 & 8.526900000 & 2.868300000 \\ 1 & 1.390200000 & 9.046800000 & 3.315800000 \\ 8 & 8.147200000 & 4.993700000 & 10.172200000 \\ 1 & 8.401200000 & 5.673600000 & 10.863900000 \\ 1 & 8.979700000 & 4.495400000 & 9.870600000 \\ 8 & -2.793500000 & -7.019700000 & 6.344000000 \\ 1 & -3.256900000 & -6.230100000 & 6.771400000 \\ 1 & -2.306700000 & -7.523200000 & 7.073800000 \\ 8 & -10.580100000 & -9.533800000 & 5.147900000 \\ 1 & -9.957800000 & -9.466100000 & 5.945300000 \\ 1 & -11.251200000 & -10.256600000 & 5.330500000 \\ 8 & -0.805900000 & -0.904600000 & -4.175800000 \\ 1 & -0.753300000 & -0.502300000 & -5.103000000 \\ 1 & -0.222600000 & -1.731900000 & -4.159500000 \\ 8 & 14.026000000 & -1.662600000 & 1.398700000 \\ 1 & 14.829500000 & -2.177600000 & 1.062700000 \\ 1 & 14.204500000 & -1.301300000 & 2.326700000 \\ 8 & 6.719400000 & 5.236500000 & 3.628300000 \\ 1 & 6.179200000 & 4.482400000 & 4.031600000 \\ 1 & 6.181600000 & 6.082200000 & 3.762100000 \\ 8 & 3.159600000 & -12.756200000 & -0.973600000 \\ 1 & 3.939300000 & -12.199300000 & -1.293900000 \\ 1 & 2.927100000 & -13.457300000 & -1.664000000 \\ 8 & 12.211400000 & -6.868900000 & 7.293200000 \\ 1 & 12.931100000 & -7.441900000 & 7.692300000 \\ 1 & 12.397600000 & -5.889500000 & 7.487600000 \\ 8 & 8.168000000 & 13.672700000 & 4.359300000\end{array}$




$\begin{array}{cccc}1 & 8.804200000 & 14.447700000 & 4.393200000 \\ 1 & 7.501300000 & 13.881000000 & 3.624200000 \\ 8 & 1.808200000 & 2.328500000 & 13.051900000 \\ 1 & 1.986900000 & 2.946800000 & 13.820900000 \\ 1 & 1.096200000 & 2.708000000 & 12.440400000 \\ 8 & -1.591100000 & 6.372600000 & 1.953800000 \\ 1 & -0.699000000 & 5.942200000 & 1.749600000 \\ 1 & -1.547600000 & 7.339600000 & 1.657500000 \\ 8 & 1.833500000 & -2.120400000 & -13.763000000 \\ 1 & 2.092000000 & -1.187200000 & -14.056100000 \\ 1 & 0.824700000 & -2.161700000 & -13.811300000 \\ 8 & 14.473000000 & -0.722000000 & -2.762400000 \\ 1 & 15.304200000 & -1.281500000 & -2.881500000 \\ 1 & 14.323100000 & -0.116800000 & -3.559200000 \\ 8 & 3.703900000 & 0.714700000 & 8.228400000 \\ 1 & 3.974500000 & 1.148700000 & 9.102800000 \\ 1 & 3.544100000 & -0.272800000 & 8.364700000 \\ 8 & -1.330100000 & -13.272300000 & -0.765300000 \\ 1 & -0.866600000 & -13.787200000 & -1.490100000 \\ 1 & -0.646900000 & -13.082000000 & -0.037600000 \\ 8 & -11.364100000 & 4.722400000 & 2.639100000 \\ 1 & -10.727300000 & 3.938500000 & 2.574700000 \\ 1 & -10.850100000 & 5.580600000 & 2.487100000 \\ 8 & 8.643600000 & -1.718900000 & 12.199700000 \\ 1 & 8.911300000 & -1.759100000 & 13.165700000 \\ 1 & 9.434400000 & -2.037200000 & 11.650800000 \\ 8 & -1.882500000 & 2.121700000 & -9.009500000 \\ 1 & -1.777900000 & 1.740000000 & -9.940800000 \\ 1 & -2.551100000 & 1.558100000 & -8.503200000 \\ 8 & -13.828200000 & -5.573100000 & 0.531200000 \\ 1 & -14.709300000 & -5.996800000 & 0.754300000 \\ 1 & -13.087500000 & -6.119700000 & 0.961900000 \\ 8 & 8.231400000 & -0.945400000 & -5.780000000 \\ 1 & 7.423800000 & -0.343700000 & -5.671200000 \\ 1 & 8.862800000 & -0.738500000 & -5.019700000 \\ 8 & -3.900000000 & 8.388100000 & -2.652000000 \\ 1 & -3.900900000 & 7.863600000 & -1.784200000 \\ 1 & -4.422600000 & 9.243700000 & -2.539600000 \\ 8 & -9.441100000 & -7.232600000 & -4.167900000 \\ 1 & -9.709500000 & -7.421100000 & -3.210300000 \\ 1 & -10.139000000 & -6.599700000 & -4.547000000 \\ 8 & 5.185400000 & 3.242800000 & 4.836500000 \\ 1 & 5.565700000 & 3.052300000 & 5.755600000 \\ 1 & 5.013700000 & 2.357100000 & 4.378000000 \\ 1 & -4.720300000 & -8.516800000 & -8.955800000 \\ 1 & -5.331400000 & -8.031500000 & -8.313100000\end{array}$




$\begin{array}{cccc}1 & -4.402700000 & -9.362100000 & -8.499800000 \\ 8 & 12.897300000 & -2.872400000 & 4.739100000 \\ 1 & 13.478000000 & -2.060900000 & 4.585200000 \\ 1 & 11.942600000 & -2.586800000 & 4.561300000 \\ 8 & 0.156600000 & 4.255200000 & 4.799400000 \\ 1 & 1.151200000 & 4.087400000 & 4.723900000 \\ 1 & 0.010600000 & 5.249300000 & 4.923000000 \\ 8 & 3.026100000 & -14.013000000 & 8.315900000 \\ 1 & 3.392300000 & -14.794100000 & 8.825000000 \\ 1 & 2.025400000 & -13.952500000 & 8.444800000 \\ 8 & -15.275600000 & 5.183100000 & 5.694200000 \\ 1 & -14.995000000 & 6.149000000 & 5.805400000 \\ 1 & -16.284800000 & 5.156600000 & 5.754700000 \\ 8 & 4.530500000 & 12.927700000 & 2.794200000 \\ 1 & 4.090600000 & 12.451000000 & 2.018600000 \\ 1 & 5.430500000 & 13.278400000 & 2.495000000 \\ 8 & -13.042200000 & -0.490300000 & 6.049400000 \\ 1 & -13.067900000 & 0.245300000 & 6.730700000 \\ 1 & -13.372200000 & -0.100400000 & 5.172300000 \\ 8 & -7.406100000 & 5.255000000 & 11.768600000 \\ 1 & -8.196400000 & 4.666800000 & 11.544500000 \\ 1 & -7.177600000 & 5.133500000 & 12.736400000 \\ 8 & 4.130200000 & -13.591400000 & 1.321700000 \\ 1 & 3.515800000 & -14.133100000 & 1.913500000 \\ 1 & 3.707600000 & -13.415700000 & 0.421300000 \\ 8 & 8.368800000 & 11.002200000 & 3.596200000 \\ 1 & 8.343500000 & 10.510400000 & 4.479800000 \\ 1 & 8.626100000 & 11.965200000 & 3.759300000 \\ 8 & 6.685500000 & -9.702300000 & 1.962000000 \\ 1 & 6.833700000 & -9.917700000 & 2.939800000 \\ 1 & 6.635400000 & -10.559100000 & 1.424100000 \\ 8 & 8.704400000 & -3.881400000 & 4.195300000 \\ 1 & 7.935200000 & -3.242100000 & 4.017900000 \\ 1 & 8.517900000 & -4.354100000 & 5.069400000 \\ 8 & -13.005100000 & -1.141900000 & -1.190000000 \\ 1 & -13.198600000 & -1.807600000 & -0.454600000 \\ 1 & -13.667200000 & -0.380200000 & -1.121700000 \\ 8 & 2.599800000 & -1.277300000 & -7.601300000 \\ 1 & 1.707400000 & -1.607300000 & -7.949200000 \\ 1 & 3.094800000 & -2.085900000 & -7.246600000 \\ 8 & -7.150800000 & 3.968400000 & 4.943600000 \\ 1 & -6.461100000 & 3.454400000 & 4.409500000 \\ 1 & -6.694600000 & 4.409800000 & 5.731000000 \\ 8 & -11.315000000 & -1.873700000 & -5.568700000 \\ 1 & -11.304900000 & -2.474900000 & -6.382000000 \\ 1 & -11.285400000 & -0.924900000 & -5.915400000\end{array}$




$\begin{array}{cccc}8 & 13.096900000 & -1.711000000 & -8.931400000 \\ 1 & 12.170700000 & -2.114200000 & -8.870400000 \\ 1 & 13.644500000 & -2.311400000 & -9.519500000 \\ 8 & 13.317500000 & -8.818000000 & 2.799100000 \\ 1 & 12.994000000 & -8.296000000 & 3.602500000 \\ 1 & 13.124700000 & -9.782300000 & 2.989900000 \\ 8 & 1.171100000 & 9.759100000 & -8.606700000 \\ 1 & 1.795800000 & 9.257100000 & -7.988400000 \\ 1 & 0.777600000 & 9.063200000 & -9.226400000 \\ 8 & -11.591500000 & 4.605900000 & 9.244400000 \\ 1 & -12.381800000 & 4.306000000 & 8.690600000 \\ 1 & -11.579700000 & 5.612200000 & 9.342200000 \\ 8 & -6.089400000 & -9.589200000 & -1.848100000 \\ 1 & -6.349800000 & -10.225000000 & -1.106100000 \\ 1 & -6.557500000 & -8.709200000 & -1.677200000 \\ 8 & 1.342600000 & 2.035000000 & -0.374300000 \\ 1 & 1.885500000 & 2.560400000 & 0.303700000 \\ 1 & 1.143500000 & 2.648900000 & -1.155400000 \\ 8 & -11.072400000 & 2.227500000 & -8.649800000 \\ 1 & -10.467300000 & 2.251900000 & -9.449100000 \\ 1 & -11.573400000 & 3.110800000 & -8.604200000 \\ 8 & 16.177300000 & -3.130300000 & 0.698000000 \\ 1 & 16.782200000 & -3.050800000 & 1.493700000 \\ 1 & 15.619200000 & -3.973700000 & 0.806600000 \\ 8 & 4.465300000 & 11.026800000 & 4.867600000 \\ 1 & 4.290900000 & 11.760700000 & 4.194200000 \\ 1 & 5.193900000 & 11.332300000 & 5.496000000 \\ 8 & 1.919500000 & -10.893600000 & 0.561100000 \\ 1 & 2.238800000 & -11.586800000 & -0.104500000 \\ 1 & 2.101700000 & -9.975200000 & 0.178100000 \\ 8 & -8.355100000 & 0.211400000 & 4.744700000 \\ 1 & -8.924000000 & -0.550100000 & 4.400700000 \\ 1 & -7.892700000 & 0.613400000 & 3.937500000 \\ 8 & -0.741000000 & -1.277300000 & 3.967700000 \\ 1 & -0.277200000 & -1.737300000 & 4.740300000 \\ 1 & -1.682700000 & -1.042100000 & 4.255800000 \\ 8 & -4.089300000 & 5.068300000 & 1.994200000 \\ 1 & -3.144800000 & 5.417700000 & 1.887700000 \\ 1 & -4.518300000 & 5.658500000 & 2.695000000 \\ 8 & -5.353700000 & -11.661200000 & -3.445800000 \\ 1 & -5.660700000 & -12.461100000 & -2.909700000 \\ 1 & -5.472900000 & -10.849700000 & -2.853800000 \\ 8 & -0.949000000 & -4.763500000 & -3.818200000 \\ 1 & -1.122400000 & -5.743600000 & -3.649500000 \\ 8 & -0.066100000 & -4.677100000 & -4.299900000 \\ & 3.299600000 & 9.011600000 & 6.234000000\end{array}$




$\begin{array}{cccc}1 & 3.092500000 & 8.304800000 & 5.540200000 \\ 1 & 3.536000000 & 9.870100000 & 5.753600000 \\ 8 & -1.849000000 & 5.552500000 & -6.870500000 \\ 1 & -1.797500000 & 6.304300000 & -7.545600000 \\ 1 & -2.819200000 & 5.401900000 & -6.620100000 \\ 8 & 6.442600000 & 8.998900000 & -6.261800000 \\ 1 & 7.285800000 & 9.307700000 & -5.790600000 \\ 1 & 6.091400000 & 9.755800000 & -6.833800000 \\ 8 & -5.669300000 & 2.771700000 & -12.113500000 \\ 1 & -6.508800000 & 2.959100000 & -12.646300000 \\ 1 & -5.767600000 & 1.833400000 & -11.748600000 \\ 8 & -8.137600000 & 0.440900000 & -0.926700000 \\ 1 & -7.528100000 & -0.366800000 & -0.888600000 \\ 1 & -9.079900000 & 0.188100000 & -1.197000000 \\ 8 & -0.633900000 & -5.234200000 & 0.911700000 \\ 1 & -1.073200000 & -4.898300000 & 1.757200000 \\ 1 & -0.581300000 & -6.244900000 & 0.921400000 \\ 8 & 7.903900000 & 9.705300000 & 6.068200000 \\ 1 & 7.169200000 & 9.046300000 & 6.291800000 \\ 1 & 7.578700000 & 10.632900000 & 6.304000000 \\ 8 & 5.017200000 & 1.086400000 & 11.108200000 \\ 1 & 5.506600000 & 0.205600000 & 11.190400000 \\ 1 & 5.669600000 & 1.778800000 & 10.754300000 \\ 8 & 10.536600000 & 1.790000000 & -8.949800000 \\ 1 & 11.420400000 & 1.575200000 & -9.389700000 \\ 1 & 9.995400000 & 0.939700000 & -8.841300000 \\ 8 & -4.315800000 & 7.601800000 & 10.120900000 \\ 1 & -4.018800000 & 8.541700000 & 9.941000000 \\ 1 & -5.319500000 & 7.584300000 & 10.248000000 \\ 8 & 12.624600000 & 5.560000000 & 3.925400000 \\ 1 & 12.584500000 & 6.013900000 & 3.024200000 \\ 1 & 12.386900000 & 6.205000000 & 4.667200000 \\ 8 & -3.908900000 & -10.818400000 & 5.557600000 \\ 1 & -3.087700000 & -10.843600000 & 4.964300000 \\ 1 & -4.719700000 & -10.564700000 & 5.006700000 \\ 8 & -7.299300000 & -7.207200000 & 1.366600000 \\ 1 & -6.306900000 & -7.340500000 & 1.504300000 \\ 1 & -7.695200000 & -6.927900000 & 2.256100000 \\ 8 & 3.453400000 & -5.983800000 & 10.378600000 \\ 1 & 3.625400000 & -6.561200000 & 11.188200000 \\ 1 & 3.990200000 & -6.366900000 & 9.613600000 \\ 8 & 1.474900000 & 11.901800000 & -4.887900000 \\ 1 & 1.754300000 & 12.716300000 & -5.418000000 \\ 8 & 2.242200000 & 11.244600000 & -4.835400000 \\ 1 & 2.612500000 & 0.021800000 & 12.126300000 \\ & 2.306200000 & 0.837500000 & 12.638200000\end{array}$




$\begin{array}{cccc}1 & 3.549400000 & 0.197400000 & 11.787200000 \\ 8 & -3.741300000 & 6.806700000 & -10.588900000 \\ 1 & -3.015800000 & 7.151400000 & -9.967100000 \\ 1 & -4.506500000 & 7.444900000 & -10.474700000 \\ 8 & -4.603600000 & -7.251400000 & 1.435200000 \\ 1 & -4.548600000 & -7.361500000 & 2.441000000 \\ 1 & -4.073600000 & -7.979800000 & 0.976200000 \\ 8 & -5.767300000 & -3.427700000 & -14.267100000 \\ 1 & -5.734000000 & -4.018600000 & -15.076800000 \\ 1 & -5.625200000 & -4.017200000 & -13.455200000 \\ 8 & 0.518700000 & -13.135900000 & 1.230500000 \\ 1 & 1.113300000 & -13.746100000 & 1.774200000 \\ 1 & 0.998600000 & -12.257800000 & 1.100400000 \\ 8 & -3.153700000 & -10.182900000 & 11.514300000 \\ 1 & -2.230100000 & -10.574400000 & 11.399300000 \\ 1 & -3.812900000 & -10.745500000 & 10.997200000 \\ 8 & 1.790600000 & -9.097100000 & 10.691400000 \\ 1 & 2.545100000 & -8.979500000 & 11.354500000 \\ 1 & 2.187800000 & -9.085400000 & 9.760200000 \\ 8 & 3.020400000 & -8.995700000 & 8.204000000 \\ 1 & 3.589200000 & -9.828800000 & 8.136500000 \\ 1 & 3.633000000 & -8.191200000 & 8.147500000 \\ 8 & 3.875300000 & -4.730400000 & 5.914200000 \\ 1 & 2.906000000 & -5.018400000 & 5.972500000 \\ 1 & 4.172800000 & -4.913700000 & 4.964500000 \\ 8 & 9.385100000 & -7.832500000 & 4.375800000 \\ 1 & 8.708600000 & -8.576400000 & 4.475700000 \\ 1 & 9.150200000 & -7.306500000 & 3.544500000 \\ 8 & 3.541800000 & 11.566800000 & 0.725100000 \\ 1 & 3.435600000 & 12.135000000 & -0.104900000 \\ 1 & 4.231500000 & 10.848600000 & 0.539600000 \\ 8 & 6.607000000 & -2.372400000 & 3.804400000 \\ 1 & 5.986700000 & -2.896100000 & 3.201700000 \\ 1 & 6.084300000 & -2.106200000 & 4.624500000 \\ 8 & 15.263300000 & 1.578000000 & 5.483200000 \\ 1 & 16.220700000 & 1.714400000 & 5.747700000 \\ 1 & 14.990200000 & 2.325200000 & 4.851300000 \\ 8 & -13.325300000 & 9.637500000 & 7.835000000 \\ 1 & -12.664400000 & 10.030000000 & 7.172900000 \\ 1 & -13.774000000 & 10.380400000 & 8.337500000 \\ 8 & 6.651600000 & -0.350000000 & -1.075400000 \\ 1 & 7.531500000 & 0.139100000 & -0.959600000 \\ & 6.564400000 & -0.557100000 & -2.062300000 \\ 1 & 9.752100000 & 4.264400000 & 1.724300000 \\ 1 & 9.558700000 & 5.083300000 & 2.284800000 \\ 1 & & \end{array}$




$\begin{array}{cccc}8 & -9.783600000 & 2.716100000 & 8.441300000 \\ 1 & -10.392100000 & 3.457900000 & 8.763500000 \\ 1 & -9.694500000 & 2.795300000 & 7.435700000 \\ 8 & -1.413200000 & 9.773400000 & -7.406300000 \\ 1 & -0.572200000 & 9.956500000 & -7.931900000 \\ 1 & -1.311700000 & 10.143800000 & -6.472500000 \\ 8 & 9.201900000 & -0.660300000 & -3.184100000 \\ 1 & 10.077700000 & -0.370900000 & -2.768100000 \\ 1 & 9.242100000 & -1.665600000 & -3.294400000 \\ 8 & 10.284300000 & 4.248600000 & -2.248700000 \\ 1 & 9.906200000 & 5.012900000 & -2.795400000 \\ 1 & 10.832000000 & 3.673000000 & -2.881900000 \\ 8 & -7.108500000 & -1.434800000 & -12.897500000 \\ 1 & -6.742200000 & -2.020900000 & -13.633600000 \\ 1 & -7.198800000 & -1.995600000 & -12.063300000 \\ 8 & 5.108600000 & 13.386400000 & -5.744900000 \\ 1 & 4.261100000 & 13.698400000 & -6.198100000 \\ 1 & 5.440000000 & 14.115400000 & -5.122000000 \\ 8 & 3.915200000 & -7.889200000 & -3.910700000 \\ 1 & 2.971300000 & -7.526200000 & -3.882700000 \\ 1 & 3.970900000 & -8.631900000 & -4.595200000 \\ 8 & -3.641700000 & 0.481500000 & -3.303300000 \\ 1 & -4.391700000 & 0.975300000 & -2.839400000 \\ 1 & -2.991200000 & 1.163500000 & -3.674400000 \\ 8 & -1.802800000 & -11.159600000 & 3.905400000 \\ 1 & -1.201200000 & -10.470700000 & 3.471800000 \\ 1 & -1.626100000 & -12.069800000 & 3.499100000 \\ 8 & -0.666700000 & -3.778300000 & -1.339500000 \\ 1 & -0.672300000 & -4.270300000 & -2.220800000 \\ 1 & -0.733400000 & -4.415200000 & -0.559400000 \\ 8 & 5.305400000 & -11.149100000 & -1.756000000 \\ 1 & 5.660000000 & -10.203900000 & -1.725700000 \\ 1 & 5.725700000 & -11.623900000 & -0.971000000 \\ 8 & -0.260300000 & 5.531700000 & 8.953500000 \\ 1 & 0.340200000 & 6.172600000 & 8.471800000 \\ 1 & -0.972000000 & 6.045000000 & 9.454500000 \\ 8 & 8.681900000 & 4.954400000 & 6.323800000 \\ 1 & 8.527100000 & 4.022400000 & 5.965800000 \\ 1 & 9.567000000 & 4.920800000 & 6.818000000 \\ 8 & 4.667900000 & 11.690100000 & -3.487700000 \\ 1 & 4.316600000 & 10.880400000 & -3.980600000 \\ 1 & 4.888400000 & 12.422600000 & -4.151000000 \\ 8 & -13.433000000 & 8.296400000 & 3.117200000 \\ 1 & -12.508100000 & 8.671900000 & 3.019900000 \\ 1 & -13.589100000 & 8.124100000 & 4.107400000 \\ 8 & -11.972000000 & 4.715400000 & -8.180500000\end{array}$




$\begin{array}{cccc}1 & -12.727200000 & 5.191100000 & -8.652900000 \\ 1 & -11.086300000 & 5.074900000 & -8.506900000 \\ 8 & 11.987200000 & -7.027300000 & 4.569800000 \\ 1 & 11.034800000 & -7.318400000 & 4.395900000 \\ 1 & 12.137100000 & -6.945100000 & 5.566000000 \\ 8 & -5.311800000 & 10.709900000 & -2.215300000 \\ 1 & -5.617000000 & 10.924500000 & -1.276400000 \\ 1 & -6.069300000 & 10.953000000 & -2.837000000 \\ 8 & -3.635500000 & -1.780000000 & -13.998200000 \\ 1 & -4.084700000 & -0.917700000 & -14.277900000 \\ 1 & -4.288500000 & -2.539200000 & -14.139700000 \\ 8 & 2.704700000 & -14.416900000 & -3.346300000 \\ 1 & 3.017800000 & -13.539600000 & -3.718000000 \\ 1 & 3.302000000 & -15.154800000 & -3.711200000 \\ 8 & -7.565400000 & 6.018900000 & 3.181300000 \\ 1 & -7.599900000 & 5.154100000 & 3.703500000 \\ 1 & -6.745500000 & 6.539900000 & 3.462200000 \\ 8 & 2.667000000 & -4.313400000 & 1.108400000 \\ 1 & 2.169300000 & -4.925100000 & 0.473400000 \\ 1 & 2.236800000 & -4.363100000 & 2.025200000 \\ 8 & -8.707900000 & -10.783300000 & 3.709200000 \\ 1 & -8.869900000 & -10.951800000 & 2.725900000 \\ 1 & -9.406300000 & -10.141900000 & 4.057800000 \\ 8 & -5.689400000 & -0.849400000 & 1.515100000 \\ 1 & -5.591200000 & -1.706900000 & 2.044000000 \\ 1 & -5.764600000 & -1.112700000 & 0.539900000 \\ 8 & 9.643500000 & 7.965200000 & -1.334000000 \\ 1 & 10.237800000 & 7.982200000 & -0.515200000 \\ 1 & 9.630100000 & 8.861300000 & -1.803900000 \\ 8 & -0.344700000 & -12.513400000 & -4.287400000 \\ 1 & -1.297100000 & -12.232200000 & -4.472400000 \\ 1 & -0.341500000 & -13.424900000 & -3.852500000 \\ 8 & 5.006200000 & 2.755300000 & -4.535100000 \\ 1 & 4.848700000 & 3.680000000 & -4.911000000 \\ 1 & 5.637600000 & 2.807800000 & -3.745800000 \\ 8 & -11.282200000 & -5.183400000 & 6.399200000 \\ 1 & -11.413500000 & -5.187200000 & 7.401000000 \\ 1 & -11.184700000 & -4.224700000 & 6.088900000 \\ 8 & 12.995000000 & 0.938600000 & -9.466900000 \\ 1 & 13.388100000 & 1.465200000 & -8.699100000 \\ 1 & 13.192000000 & -0.039100000 & -9.304300000 \\ 8 & -2.850100000 & 11.600700000 & -2.678900000 \\ 1 & -2.687200000 & 12.549000000 & -2.395700000 \\ 1 & -3.777400000 & 11.330500000 & -2.369500000 \\ 8 & -5.744400000 & -8.025600000 & 8.096600000 \\ 1 & -5.056300000 & -8.733600000 & 7.877600000\end{array}$




$\begin{array}{crrc}1 & -5.866100000 & -7.970700000 & 9.101500000 \\ 8 & 6.568000000 & -8.701200000 & 11.091000000 \\ 1 & 7.286900000 & -9.328500000 & 11.396300000 \\ 1 & 6.774200000 & -8.436800000 & 10.137000000 \\ 8 & -9.648100000 & -8.358600000 & 0.818900000 \\ 1 & -8.731600000 & -8.018800000 & 1.077000000 \\ 1 & -9.622300000 & -9.366300000 & 0.744400000 \\ 8 & -2.917500000 & -4.831400000 & -10.785300000 \\ 1 & -2.080300000 & -4.504400000 & -11.244700000 \\ 1 & -2.653100000 & -5.719400000 & -10.383500000 \\ 8 & -6.799700000 & -5.754500000 & 11.363500000 \\ 1 & -6.564900000 & -6.420100000 & 12.085200000 \\ 1 & -7.701000000 & -6.005400000 & 10.982300000 \\ 8 & -7.073400000 & 7.753900000 & -4.372400000 \\ 1 & -7.554200000 & 7.145900000 & -5.023800000 \\ 1 & -6.186500000 & 7.987500000 & -4.795700000 \\ 8 & -5.588800000 & 0.222300000 & -11.432400000 \\ 1 & -6.157900000 & -0.431900000 & -11.952400000 \\ 1 & -4.647400000 & -0.142800000 & -11.361400000\end{array}$

